\title{
Social Capital and Entrepreneurial Performance in Russia: A Panel Study
}

\author{
By: Bat Batjargal
}

Working Paper Number 352

December 2000 


\title{
SOCIAL CAPITAL AND ENTREPRENEURIAL PERFORMANCE IN RUSSIA:
} A PANEL STUDY

\author{
BAT BATJARGAL ${ }^{*}$ \\ Davis Center for Russian Studies \\ Harvard University \\ 1737 Cambridge Street \\ Cambridge, MA 02138 \\ Guanghua School of Management \\ Peking University \\ Beijing, 100871 \\ People's Republic of China \\ Tel: 861062757763
}

Email: bbat@gsm.pku.edu.cn

\footnotetext{
* I am grateful to the International Research \& Exchange Board (IREX) for its financial support for this research. IREX is not responsible for the views expressed herein. I would like to thank seminar participants at the Davis Center for Russian Studies at Harvard University, Center for International Development at Harvard University and London Business School for constructive comments on earlier versions of the paper.
} 


\title{
SOCIAL CAPITAL AND ENTREPRENEURIAL PERFORMANCE IN RUSSIA: A PANEL STUDY
}

\begin{abstract}
Drawing on the social embeddedness perspective, this paper examines the impact of entrepreneurs' social capital on their firm performance in post-Soviet Russia. Based on face-to-face interviews with 75 Russian entrepreneurs in 1995, and the follow-up study in 1999, the study contrasts the effects of structural embeddedness, relational embeddedness and resource embeddedness on firm performance. The main finding is that relational embeddedness and resource embeddedness have direct positive impacts on sales growth, profit margin and return on assets in contrast to structural embeddedness that has no impact on performance. The research implies that further research should focus on finding out what dimensions of social capital affect what performance indicators and how they affect. The practical implication is that entrepreneurs should recruit more resource-rich weak ties into their personal networks.
\end{abstract}

KEY WORDS: social capital, firm performance, Russia 


\section{INTRODUCTION}

Economic performance of firms in transition economies has been explained by ownership category, industry, and geographic region (Earle, Estrin \& Leshchenko, 1996, Richter \& Schaffer, 1996), start-up capital and previous private sector experience of the entrepreneur (Johnson \& Loveman, 1995). In this study, I examine the impact of entrepreneurs' social capital on firm performance and suggest a socialized explanation of company performance.

In his seminal essay, Granovetter (1985) postulated that actions of economic agents are embedded in concrete, ongoing systems of social relations and these relations facilitate and constrain agents' profit and rent seeking actions. Although there are recent successful attempts to operationalize and empirically test the exchange process of embedded actions (Uzzi, 1996, 1997, 1999), the bulk of the empirical research has been devoted to the aspect of instrumental utilisation of personal relations and networks by corporate and individual actors (Portes, 1998).

Social capital defined as networks of relationships and assets located in these networks (Bourdieu, 1986, Burt, 1997a, Coleman, 1988) has been found positively influencing firm performance (Baker, 1990), product innovation (Tsai \& Ghoshal, 1998), and industry-wide network formation (Walker, Kogut \& Shan, 1997). Similarly, social capital of individuals facilitates job and status attainment (Lin, Ensel, \& Vaughn, 1981, Marsden \& Hurlbert, 1988), enhances individual's power (Krackhardt, 1990) and career mobility (Podolny \& Baron, 1997), and impacts CEO compensation (Belliveau, O’Reilly III, \& Wade, 1996). 
Research on personal networks of entrepreneurs revealed that entrepreneurs perceive and exploit business opportunities in disconnected networks (Burt, 1992), seek information, advice and social support from network alters (Aldrich \& Zimmer, 1986, Birley, 1985, Nohria, 1992), control and manage exchange structures through network dyads (Larson, 1992), access financial capital (Shane \& Cable, 1999), and get an endorsement from prestigious players to influence perceptions of the quality of their ventures (Stuart, Hoang, \& Hybels, 1999).

Drawing on the embeddedness perspective and social network theory, I attempt to link varieties of firm performance with initial social networks of entrepreneurs in the context of a transition economy - the Russian Federation. I argue that different dimensions of social capital, e.g., structural composition, relational content and resources, have varying effects on company performance confirming Granovetter's idea of differentiated influences of various types of embeddedness on individuals' economic action (Granovetter, 1990: 99).

I seek to make two contributions to the research literature: First, by applying the embeddedness argument and social capital theory developed and tested in the Western social environment to the Russian reality, I expand the paradigm boundary to formerly communist societies. Second, in addition and contrast to the previous research, I demonstrate the varying utilitarian value of different components of social capital for several performance indicators and establish a ranking order of their instrumental usefulness.

Resources of individuals defined as valuable assets possessed by persons and embedded in a social network constitute the fundamental linkage between actors' 
purposive actions and their outcomes, e.g., entrepreneurial entry and exit (Abell, 1996). Initial resources of entrepreneurs, therefore, are found to have a lasting impact upon the venture's performance (Brush \& Chaganti, 1999, Cooper, Gimeno-Gascon, \& Woo, 1994, Eisenhardt \& Schoonhoven, 1990). However, the pattern of dispersion of various resources among individuals at a given moment in time is a function of social structure (Stinchcombe, 1965). Different resources or capital are dispersed unevenly across the hierarchical as well as segmented groups in society (Anheier, Gerhards \& Romo, 1995). The volume of resources possessed by an individual or groups is contingent on the overall position of that individual or group in the social space. This uneven resource dispersion across social groups and individuals is referred to resource heterogeneity of social actors. The resource heterogeneity forms the set of constraints that governs the functioning of society in a durable way determining the chances of success for instrumental actions of individuals (Bourdieu, 1986).

Social capital heterogeneity of entrepreneurs refers to the uneven endowment of entrepreneurs with social resources in terms of network range (Burt, 1983b), relations and contact resources (Lai, Lin \& Leung, 1998). Social capital heterogeneity of entrepreneurs leads to varieties of firm performance because social relations flavour purchase and sale decisions of entrepreneurs.

Sociologists elaborated three dimensions of individual's social capital: structural embeddedness - the structure of the overall network of relations, relational embeddedness - the extent to which economic actions are affected by the quality of actors' personal relations (Granovetter, 1990: 98), and cognitive embeddedness - the degree to which an individual shares common code and systems of meaning with a community or collective 
(Nahapiet \& Ghoshal, 1998: 244). Fourth dimension of social capital may be articulated as resource embeddedness - the degree to which network ties contain valuable instrumental resources (Lai, et al, 1998, Lin \& Dumin, 1986, Marsden \& Hurlbert, 1988). The resource embeddedness has been referred as "the material quality of ties" (Uzzi, 1996: 675), and it is a function of attributes and characteristics of individual alters, e.g., high status contact versus low status contact (Ibarra, 1993). In this study, I focus upon structural embeddedness, relational embeddedness and resource embeddedness of entrepreneurs' social capital.

I operationalize structural embeddedness as network size and heterophily. Network size is defined as the number of direct ties involving individual units (Burt, 1983b, Marsden, 1990). Network heterophily refers to the degree which an ego network contains diverse alters, e.g., demographic characteristics or occupational status (Burt, 1983b, Ibarra, 1993, Marsden, 1987, Renzulli, Aldrich \& Moody, 1999).

Relational embeddedness has been interpreted as relational content (Burt, 1983a, 1997b, Podolny \& Baron, 1997), tie strength (Marsden \& Campbell, 1984), and relational trust (Galunic \& Moran, 1999, Tsai \& Ghoshal, 1998). I operationalize relational embeddedness as "the friendship domain" indicating strong ties and "the acquaintance domain" indicating weak ties (Burt, 1983a, Krackhardt, 1992, Lin \& Dumin, 1986).

Lai et al (1998) operationalized resource embeddedness as contact's resource characteristics that are contingent upon occupational status, authority position, and core versus peripheral sector. I operationalize resource embeddedness as the extent to which an egocentric network is comprised of actors of high socioeconomic status and the extent 
to which entrepreneurs have been able to marshal financial resources from their personal networks.

\section{THE RUSSIAN CONTEXT}

The instrumental use of personal connections characterized Soviet society at its core. Blat - the system of informal contacts served as an alternative social mechanism for overcoming rigidities in Soviet factory's production and supply practices (Berliner, 1957) and obtaining consumer goods and services under the rationing system that characterized the Soviet economy (Ledeneva, 1998). The scale of informal ties and resources located in these networks were dependent on the political power of social actors (Shkaratan \& Figatner, 1992). Vladimir Yadov, a leading Russian sociologist, stated in an interview: "Russian entrepreneurship is embedded in neither markets nor hierarchy but in a hybrid form of the two. There exist, therefore, parallel structures where the same amount of money and connections have different values in each. You can obtain the same volume of profit by spending different amounts of money. Succeeding in Russian capitalism depends not only on your financial capacity but also on many other factors such as informal networks, trust, etc. (Author's interview, May 1995, Moscow). This may indicate that social relations have profound effects on business transactions in the transition economy of Russia because of the unstable institutional and deficient regulatory framework (Sedaitis, 1998, Stark, 1996).

Based upon the logic of social embeddedness and social capital heterogeneity of entrepreneurs, I propose a number of empirical hypotheses. 


\section{HYPOTHESES}

\section{Structural Embeddedness}

Network Size. It is assumed that extensive personal networks of entrepreneurs may increase the likelihood of locating clients and suppliers who are socially bound. This may facilitate sales stabilization and eventual growth since the embeddedness provides a flexible room for negotiations that might allow entrepreneurs to convert the social bonds into revenue growth and other tangible benefits. The personal chemistry between the entrepreneur and the supplier is likely to enable the entrepreneur to purchase raw materials and other production inputs at lower prices, and that might influence profit margin boosting the overall performance. There are empirical evidence that personalized relations between entrepreneurs and their bankers lead to cheaper interest rates on loans (Uzzi, 1999), and spin-off firms get favourable rates on equipment leasing from their parent companies (Webster \& Charap, 1993). These arrangements may improve firm's financial performance ratios such as return on assets.

Network Heterophily. Heterophilous networks are conducive to interactions of entrepreneurs with diverse others of different attributes. Bankers may be able to build a broad range of clients' base with differentiated needs across different industries, and this may enable them to customize their products building customer loyalty and spread risks

of defaults. In this way, bankers are likely to build customers' dependence that may enhance their client retention and wallet penetration capabilities. Trading firms may get better access to overdraft facilities, speedy cash management and other services from embedded relationships with bankers whereas production firms may deliver goods in time and be flexible to ad hoc customer demands. The network diversity may be crucial 
for manufacturing firms in the Russian condition: the wide-spread phenomenon of interenterprise loans, enterprise arrears for delivered products and barter exchanges that plagued the Russian economy ever since the late 1980s (Dolgopyatova, 1995) may have forced Russian manufacturers to diversify their networks in order to survive. The available empirical evidence supports the proposition that firms in transition economies enter and build deliberately a complicated web of interconnected firms where assets and liabilities are creatively dispersed in order to reduce the harming effects of environmental uncertainties (Stark, 1996). The simple production chain of resource firms makes them less reliant on personal relations with managers of trading or manufacturing firms although they are likely to gain benefits from bankers in services such as foreign currency exchange or international money transfer and etc.

The empirical research on effects of structural elements of networks on business performance produced mixed evidence. Reese \& Aldrich (1995) found no evidence to suggest that the size of an entrepreneur's personal network affects venture survival. Aldrich et al documented that network density was associated with profitability of new ventures whereas network accessibility was positively correlated with business founding (Aldrich, Rosen and Woodward, 1987). In a study of sales managers in a Fortune 100 firm, Galunic \& Moran (1999) found that network size was positively associated with sales whereas network density was negatively correlated with performance although the latter was not statistically significant.

The vital role of social networks in Russian entrepreneurship has been widely reported although most of the evidence researchers displayed remain descriptive (Huber \& Worgotter, 1998). Sedaitis (1998) found that low dense networks of start-up 
entrepreneurs were associated with better sales records in contrast to high-density networks of spin-off entrepreneurs in Russia.

H1a. The greater the size of initial networks of entrepreneurs, the better the firm's performance.

H1b. The greater the heterophily of initial networks of entrepreneurs the better the firm's performance.

\section{Relational Embeddedness}

Strong and Weak Ties. In contrast to structural embeddedness, Granovetter (1990) stated, relational embeddedness has typically quite direct effects on individual economic actions. Constrained and facilitated by a history of interactions and consequent mutual expectations, actions of economic agents such as price negotiation is largely a function of the personal chemistry as much as rational cost-benefit calculations. For example, Baker (1984) found that the increase in number of option traders impeded communication among actors and that resulted in option price volatility. This happened because as group size increased, the number of personalized trading relations that the average trader could sustain did not. In this way, relational quality preconditioned option price stability that affects directly firm or trader performance.

Strong ties are described to enhance firm performance directly through trust building, information transfer, and joint problem solving arrangements (Uzzi, 1997). Weak ties also are regarded as performance boosting devices: vaguely defined relationships provide that crucial freedom to act upon opportunities and entrepreneurs with structural autonomy are likely to gain most being not bound by expectations and obligations (Burt, 1992). 
There is no empirical study to date that examines effects of strong (friendship) and weak (acquaintance) ties on entrepreneurial performance. There is, however, a finding that friendship ties are more instrumental in finding better jobs (Lin \& Dumin, 1986). Relational trust and closeness as indicators of relational quality have been found significantly related to managerial sales and innovation performance (Galunic \& Moran, 1999). Ethnographic evidence from Russia suggest that friendship and acquaintance as exchange modes are becoming more and more utility maximizing channels (Ledeneva, 1998).

H2a. The greater the number of strong ties in initial networks of entrepreneurs the better the firm's performance.

$H 2 b$. The greater the number of weak ties in initial networks of entrepreneurs the better the firm's performance.

\section{Resource Embeddedness}

Network Resourcefulness. Personal networks that are composed of resourceful and powerful ties will produce higher rates of returns when they are utilized. Burt wrote: "a person with a poorly structured network that includes just one well-placed contact can do well through that contact's sponsorship regardless of how well the person's network as a whole is structured (Burt, 1992: 272). Connections with executives that manage large corporations and banks are conducive to greater volumes of material resources and lucrative contracts. Powerful bureaucrats will be able to provide such "services" as state contracts, tax release and other tangible benefits for firms. Bureaucratic services are

prevalent in Russia where political power has a definite market value (Kryshtanovskaya \& White, 1996). A meager empirical research on contact resources i.e., wealth, status and 
power, found that both the availability of resources and the actual mobilization of resources played a crucial role in finding better jobs (Lai et al, 1998).

H3a. The greater the resourcefulness of initial networks of entrepreneurs the better the firm's performance.

$H 3 b$. The greater the resources mobilized from initial networks the better the firm's performance.

\section{METHODOLOGY}

\section{Sample and Data Collection}

The empirical data of the study is composed of the face-to-face interviews with 75 Russian entrepreneurs in February-June 1995, and the follow-up interviews with 56 original respondents in March-May 1999. Pilot interviews with six Moscow firms were conducted in August 1994.

In 1995, I selected firms on the basis of a stratified random sampling procedure in three Russian cities, viz., Moscow, Ekaterinburg and Petrozavodsk. The computerised database of registered businesses of the Moscow City Committee of Statistics, Business Assistance Centre of the Sverdlovsk Regional Administration in Ekaterinburg, and the State Committee of Statistics of the Republic of Karelia in Petrozavodsk were used as sampling populations. I created twelve lists of firms (four industries and three sizes) each of which contained twenty firm names.

Banks were classified in accordance with the following criteria: small charter funds < US\$50 000, medium charter funds US\$50 001-250 000, and large charter funds > US\$250 001. This grouping was confirmed in interviews with Russian experts and Central bank officials. A similar classification has been established in another study of Russian banks (Lapidus \& van de Waal-Palms, 1997). In manufacturing and the resource 
sector, firms were grouped: small $-<100$ employees, medium - 101-500 employees, and large - > 501 employees. Trade firms were classified: small - < 50 employees, medium 51-200 employees, and large - > 201 employees. The classification is based on the definition of small firms in the Russian law and discussions with Russian experts (Rossiiskaya federatsiya, 1995).

Every second firm on these lists was selected for contact. In 1995, 120 entrepreneurs were contacted and 82 agreed to be interviewed. The response rate was 68 percent. 7 respondents were discovered as ineligible in the field, so that the final sample consisted of 75 entrepreneurs and directors. There were 50 new ventures or de novo firms and 25 privatized companies. Interviews were conducted with a specially designed questionnaire that contained questions on entrepreneurial networks and firm characteristics. The follow-up study in 1999 concentrated on network dynamics and firm performance. I re-interviewed 56 original respondents.

\section{Insert Tables 1 and 2 about here}

Financial data was collected from firms as well as other sources such as the Central Bank of Russia, Association of Russian Banks, the Foundation for Small Business Development in three cities, and local tax offices. About a half of the sample firms provided annual reports that contained accounting information. In most occasions, financial directors or chief accountants were interviewed additionally on financial issues. Accounting data in Roubles have been deflated by the year's average exchange rate of US\$ and Russian Roubles published in The Economist. The reliability and consistency of company financial statements still remains questionable in Russia although significant 
progress has been made for the last few years to bring Russian accounting practices in the line with the Western standard.

\section{Dependent Variable}

The dependent variable is firm performance. Organisational performance may be measured in various ways (March \& Sutton, 1997, Meyer, 1994). In this paper, firm performance is measured by sales growth, operating profit margin and return on assets (Earle et al, 1996). Sales growth for each year (1996, 1997, 1998), and the average sales growth for three years were expressed in percentage. I use sales growth rather than sales figures because of the mixed sample of large, medium and small firms as well as firms from four different industries. Operating profit margin, average operating profit margin, return on assets and average return on assets were expressed in percentage.

\section{Independent Variables}

Independent variables are network size, network heterophily, strong ties, weak ties, network resourcefulness and financial resources mobilized 95.

Network size is measured by the number of ties indicated by entrepreneurs. I presented a table where twelve types of occupations (high rank official in ministries and agencies, middle and low rank official in ministries and agencies, high rank official in

local governments, middle and low rank official in local governments, managers of large banks, managers of medium and small banks, managers of large manufacturing plants, managers of medium and small manufacturing plants, managers of large trade firms, managers of medium and small trade firms, managers large resource sector firms, and managers of medium and small resource sector firms) were listed in rows, and two types 
of tie strength (friendship and acquaintances) were placed in columns (Lin \& Dumin, 1986). I asked the respondents to indicate how many people were in each cell.

Network heterophily measures the degree to which an egocentric network contains alters from industries other than the respondent's own occupational background. Heterophily captures the proportion of non-industry contacts.

Strong ties are the number of "friends". Weak ties measure the number of "acquaintances". I presented the Russian translations: "drug" as friend and "znakomyi" as acquaintance due to the culture sensitive nature of concepts "friendship" and "acquaintance".

Network resourcefulness captures the number of high rank officials in ministries, high rank officials in local governments, managers of large banks, managers of large manufacturing plants, managers of large trade firms, and managers of large resource sector firms. Financial resources mobilized 95 is a binary variable of one if resources were mobilized before the interview and zero if resources were not mobilized.

\section{Control Variables}

Control variables are industry (banking, trade, manufacturing, and the resource sector), firm size (large, medium and small), region (Moscow, Ekaterinburg, and Petrozavodsk) and firm origin (new venture versus privatized). Three cities represent Russia's regional economic diversity well. Moscow is the financial and commercial center whereas Ekaterinburg is famous for its heavy industry. The timber industry and trade is highly developed in Petrozavodsk, the capital city of the Republic of Karelia. All controls were turned into dummies and included in the regression analysis. 


\section{RESULTS}

\section{Descriptive Statistics}

The mean performance indicators given in Table 3 show that Russian firms performed poorly: average sales growth for three years was $1 \%($ s.d. $=0.33)$, average profit margin was $-22 \%$ (s.d. $=0.86)$, and average return on assets was $-9 \%($ s.d. $=$ 0.96).

The mean network size was 34 persons $($ s.d. $=9.42)$ and the mean heterophily was $82 \%$ (s.d. = 0.06). A typical entrepreneur had 12 friends (s.d. = 4.7) and 22 acquaintances $($ s.d. $=6.5)$. The mean of resource-rich ties was $16($ s.d. $=6.6)$, and 41 percent of interviewees $($ s.d. $=0.49)$ mobilized financial resources.

\section{Firm Performance}

Based on significant correlation coefficients, I included weak ties and resources mobilized 95 as independent variables and sales growth 98, profit margin 98, return on assets 98 and average sales growth as dependent variables in the regression analysis.

Table 4 provides the results of multivariate regression predicting firm performance as a function of social capital of entrepreneurs, controlling for industry, firm size, firm origin and region. The baseline model (1) includes industry, size, firm origin and region dummies. Resource sector dummy, Petrozavodsk dummy, medium size dummy, and privatised dummy were excluded in the regression analysis due to their weak contributions to the models. Models 2-7 show the effects of two independent variables on various performance indicators. Model 2 demonstrates that weak ties $(\mathrm{B}=$ $0.02, \mathrm{p}<0.05)$ are positively related to sales growth 98 although the model is not significant. Model 3 indicates that resources mobilized $95(\mathrm{~B}=0.35, \mathrm{p}<0.01)$ are 
associated with sales growth 98 and the model is significant $\left(F=2.11, \mathrm{R}^{2}=0.25\right)$. Model 4 reports that weak ties $(B=0.01, p<0.05)$ and resources mobilized $95(B=0.37, p<0.01)$ both are positively and significantly correlated to sales growth 98 . The overall model is also significant $\left(\mathrm{F}=3.50, \mathrm{R}^{2}=0.39\right)$. Models 5 and 6 reveal that weak ties are positively correlated to profit margin $98(\mathrm{~B}=0.05, \mathrm{p}<0.05)$ and return on assets $98(\mathrm{~B}=0.05$, $\mathrm{p}<0.05)$. None of the models is significant. Model 7 shows that resources mobilized 95 $(B=0.26, p<0.01)$ are associated with average sales growth, and the model $\left(F=1.97, R^{2}\right.$ $=0.17)$ is significant.

\section{DISCUSSION}

Pearson's correlations presented in Table 3 confirm that no indicator of structural dimension of entrepreneurs' social capital is associated with firm performance variables. This means that the proposed hypotheses on network size (H1a) and heterophily (H1b) are not affirmative. The finding is consistent with Granovetter's (1990) conclusion that structural embeddedness does not affect directly individuals' economic actions. The heterogeneity in structural characteristics of social capital does not lead to differentiated performance of Russian entrepreneurial firms.

While strong ties (friendship) do not facilitate better performance, weak ties (acquaintance) do influence positively firm's sales growth, profit margin and return on assets. Hypothesis $2 \mathrm{a}$ is not supported whereas hypothesis $2 \mathrm{~b}$ is confirmed. The finding is in the line to confirm tentatively the proposition that relational embeddedness directly impacts economic actions. The initial heterogeneity in relational dimension of social capital is conducive to diverse performance outcomes. 
The empirical evidence rejects the hypothesis on resourcefulness (H3a), i.e., the availability of resources, but supports the hypothesis on resources mobilized $95(\mathrm{H} 3 \mathrm{~b})$, i.e., the actual utilization of resources. The fact confirms the relative explanatory power of resource embeddedness as a dimension of social capital. In contrast to weak ties that had affected all three performance indicators, resources mobilized 95 have boosted sales growth 98 and average sales growth. This indicates that different aspects of social capital influence different performance indicators. Overall, varieties in performance parameters are the result of the resource heterogeneity of social networks of Russian entrepreneurs.

The striking finding is that weak ties and resources are useful in the period of economic crisis: two variables are significant in 1998, the year of the Russian default. It may be tentatively concluded that social capital is particularly valuable in the condition of extreme uncertainty.

\section{CONCLUSION}

Various dimensions of individuals' social capital have different effects on entrepreneurial performance in Russia: Relational embeddedness and resource embeddedness have direct positive impacts on sales growth, profit margin and return on assets in contrast to structural embeddedness that has no impact on performance. The heterogeneity in relational and resource dimensions of initial social capital of entrepreneurs, therefore, determines varieties of entrepreneurial performance in Russia.

The study leads to the conclusion that the basic assumptions of embeddedness perspective and social capital theory pertains in the Russian social and economic conditions. 
Several limitations should be emphasized. The sample size is relatively small, and therefore, one should be cautious of over-generalization of the results. The research site is Russia, which is going through the simultaneous social, economic and political crises, and therefore, this limits the generalizability of the findings to more stable Western societies. Company performance influencing variables such as strategy or entrepreneurial human capital were not incorporated in the model, and therefore, the study may have over-emphasized the effects of social capital.

The research implies that further research should focus on finding out what dimensions of social capital affect what performance indicators, and how they affect. The practical implication is that entrepreneurs should recruit more resource-rich weak ties into their personal networks. 


\section{REFERENCES}

Abell, P. (1996) "Self-employment and entrepreneurship. A study of entry and exit." James Coleman ed. J. Clark, 175-206 London: Falmer Press.

Aldrich, H. \& Zimmer, C. (1986) "Entrepreneurship through social networks." In The art and science of entrepreneurship ed. D. Sexton and R. Smilor. 3-23. Cambridge, Mass: Ballinger Publishing.

Aldrich, H, Rosen, B, \& Woodward, W, (1987) "The impact of social networks on business foundings and profit: A longitudinal study." In Frontiers in entrepreneurship research: ed. J. Churchil et al, 154-168. Wellesley, Mass: Babson College.

Anheier, H, Gerhards, J, \& Romo, F, (1995) "Forms of capital and social structure in cultural fields: Examining Bourdieu's social topography." American Journal of Sociology, 100 (4): 859-903.

Baker, W. (1984) "The social structure of a national securities market", American Journal of Sociology, 89 (4): 775-811.

Baker, W. (1990) "Market networks and corporate behavior." American Journal of Sociology, 96 (3): 589-625.

Belliveau, M, O'Reilly, C, \& Wade, J (1996) "Social capital at the top: Effects of social similarity and status on CEO compensation." Academy of Management Journal, 39(6): 1568-1593.

Berliner, J. (1957) Factory and manager in the USSR, Cambridge, MA: Harvard University Press.

Birley, S. (1985) "The role of networks in the entrepreneurial process." Journal of Business Venturing, 1(1): 107-117.

Bourdieu, P. (1986) "The forms of capital. Handbook of theory and research for the sociology of education 241-258. New York: Greenwood.

Brush, C. \& Chaganti, R. (1999) "Businesses without glamour? An analysis of resources on performance by size and age in small service and retail firm." Journal of Business Venturing, 14: 233-257.

Burt, R. (1983a) “Distinguishing relational contents." Applied network analysis, A methodological introduction ed. R. Burt and M. Minor. 35-74. London: Sage.

Burt, R. (1983b) Range. Applied network analysis, A methodological introduction ed. R. Burt and M. Minor. 176-194 London: Sage. 
Burt, R. (1992) Structural holes, The social structure of competition, Cambridge: Harvard University Press

Burt, R. (1997a) “The contingent value of social capital.” Administrative Science Quarterly, 42: 339-365.

Burt, R. (1997b) "A note on social capital and network content." Social Networks, 19: 355-373.

Coleman, J. (1988) "Social capital in the creation of human capital." American Journal of Sociology, 94: S95-S120.

Cooper, A, Gimeno-Gascon, J, \& Woo, C. (1994) "Initial human and financial capital as predictors of new venture performance.” Journal of Business Venturing, 9: 371-395.

Dolgopyatova, T. (1995) Rossiiskie predpriyatiya v perekhodnoi ekonomike, ekonomicheskie problemy i povedenie, Moscow: Delo.

Earle, J, Estrin, S, \& Leshchenko, L, (1996) “Ownership structures, patterns of control, and enterprise behaviour in Russia." In Enterprise restructuring and economic policy in Russia ed. S. Commander, Q. Fan \& M. Schaffer, 205-252 Washington, DC: the World Bank.

Eisenhardt, K. \& Schoonhoven, C. (1990) "Organizational growth: linking founding teams, strategy, environment, and growth among U.S. semi-conductor ventures." Administrative Science Quarterly, 28: 274-291.

Galunic, C. \& Moran, P. (1999) Social capital and productive exchange: Structural and relational embeddedness and managerial performance link. manuscript, London Business School

Granovetter, M. (1985) "Economic action and social structure: The problem of embeddedness.” American Journal of Sociology, 91 (3): 481-510.

Granovetter, M. (1990) "The old and the new economic sociology: A history and an agenda."Beyond the market place: Rethinking economy and society ed. R. Friedland \& A. Robertson, 89-112. New York: Walter de Gruyter.

Huber, P. \& Worgotter, A. (1998) “Observations on Russian business networks.” PostSoviet Affairs, 14: 81-91.

Ibarra, H. (1993) "Personal networks of women and minorities in management: a conceptual framework." Academy of Management Review, 18 (1): 56-87

Johnson, S. \& Loveman, G. (1995) Starting over in Eastern Europe: Entrepreneurship and economic renewal, Boston: Harvard Business School Press. 
Krackhardt, D. (1990) "Assessing the political landscape: structure, cognition, and power in organizations.” Administrative Science Quarterly, 35: 342-369.

Krackhardt, D. (1992) "The strength of strong ties: The importance of philos in organizations." In Networks and organizations: Structure, form, and action ed. N. Nohria \& R. Eccles. 216-239. Cambridge, Mass: Harvard Business School Press.

Kryshtanovskaya, O. \& White, S. 1996. From Soviet nomenklatura to Russian elite. Europe-Asia Studies, 48 (5): 711-733.

Lai, G. Lin, N. \& Leung, S. (1998) "Network resources, contact resources and status attainment." Social Networks, 20: 159-178.

Lapidus, M. \& van de Waal-Palms, P. (1997) Understanding Russian banking, Moscow: Mir.

Larson, A. (1992) "Network dyads in entrepreneurial settings: A study of the governance of exchange relationships." Administrative Science Quarterly, 37: 76-104.

Ledeneva, A (1998) Russia's economy of favours: Blat, networking and informal exchange, Cambridge: Cambridge University Press.

Lin, N. \& Dumin, M. (1986) “Access to occupations through social ties." Social Networks, 8: 365-385.

Lin, N, Vaughn, J. \& Ensel, W. (1981) "Social resources and occupational status attainment." Social Forces, 59(4): 1163-81.

March J. \& Sutton, R. 1997. "Organizational performance as a dependent variable." Organisation Science, 8 (6): 698-706.

Marsden, P (1987) "Core discussion networks of Americans.” American Sociological Review, 52: 122-131.

Marsden, P. (1990) "Network data and measurement." Annual Review of Sociology, 16: 435-463.

Marsden, P. \& Campbell, K. (1984) “Measuring tie strength.” Social Forces, 63 (2): 482-501.

Marsden, P. \& Hurlbert, J. (1988) "Social resources and mobility outcomes: A replication and extension." Social Forces, 67: 1038-1059. 
Meyer, M. 1994. "Measuring performance in economic organisations". In The Handbook of Economic Sociology ed. N. Smelser and R. Swedberg, 556-578, Princeton: Princeton University Press.

Nahapiet, J. \& Ghoshal, S. (1998) "Social capital, intellectual capital, and organizational advantage." Academy of Management Review, 23 (2): 242-266.

Nohria, N. (1992) "Information and search in the creation on new business ventures: the case of the 128 venture group, In Networks and organizations: Structure, form, and action, ed. N. Nohria \& R. Eccles, 240-261 Boston: Harvard Business School

Podolny, J. \& Baron, J. (1997) "Resources and relationships: social networks and mobility in the work place." American Sociological Review, 62: 673-693.

Portes, A (1998) "Social capital: Its origin and applications in modern sociology." Annual Review of Sociology, 24: 1-24.

Reese, P \& Aldrich, H. (1995) "Entrepreneurial networks and business performance: A panel study of small and medium-sized firms in the research triangle."In International Entrepreneurship ed. S. Birley, and I. Macmillan, 124-146 London: Routledge.

Renzulli, L. Aldrich, H. \& Moody, J. (1999) Family matters: Gender, networks, and entrepreneurial outcomes, Manuscript. Department of Sociology, University of North Carolina at Chapel Hill.

Richter, A. \& Schaffer, M. (1996) "The performance of de novo private firms in Russian manufacturing." Enterprise restructuring and economic policy in Russia ed. S.

Commander, Q. Fan, and M. Schaffer, 253-274 Washington, DC: the World Bank.

Rossiiskaya federatsiya. (1995) Zakon o gosudarstvennoi podderzhke malogo predprinimatel'stva, Moscow.

Sedaitis, J. (1998) “The alliances of spin-offs versus start-ups: Social ties in the genesis of post-Soviet alliances" Organization Science, 9 (3): 368-381.

Shane, S. \& Cable, D. (1999) Social capital and the financing of new ventures, manuscript, MIT Sloan School of Management.

Shkaratan, O. \& Figatner, Yu. (1992) "Starye i novye khozyaeva Rossii." Mir Rossii, 1(1): 67-90.

Stark, D. (1996) "Recombinant property in East European capitalism." American Journal of Sociology, 101 (1): 993-1027.

Stinchcombe, A. (1965) "Social structure and organisations"in Handbook of organisations ed. J. March. 142-193 Chicago: Rand McNally. 
Stuart, T. Hoang, H. \& Hybels, R. (1999) "Interorganizational endorsement and the performance of entrepreneurial ventures." Administrative Science Quarterly, 44:315-349.

Tsai, W. \& Ghoshal, S. 1998. "Social capital and value creation: The role of intrafirm networks." Academy of Management Journal, 41 (4): 464-476.

Uzzi, B. (1996) "The sources and consequences of embeddedness for the economic performance of organisations: The network effect." American Sociological Review, 61: 674-698.

Uzzi, B. (1997) "Social structure and competition in interfirm networks: The paradox of embeddedness." Administrative Science Quarterly, 42: 35-67.

Uzzi, B. (1999) "Embeddedness in the making of financial capital: How social relations and networks benefit firms seeking financing." American Sociological Review, 64: 481505.

Walker, G. Kogut, B. Shan, W. (1997) "Social capital, structural holes, and the formation of an industry network." Organization Science, 8(2): 109-125.

Webster, L. \& Charap, J.(1993) The emergence of private sector manufacturing in St. Petersburg, Technical paper no. 228, Washington D. C: the World Bank. 


\section{TABLE 1}

The Number of Entrepreneurs Interviewed in 1995

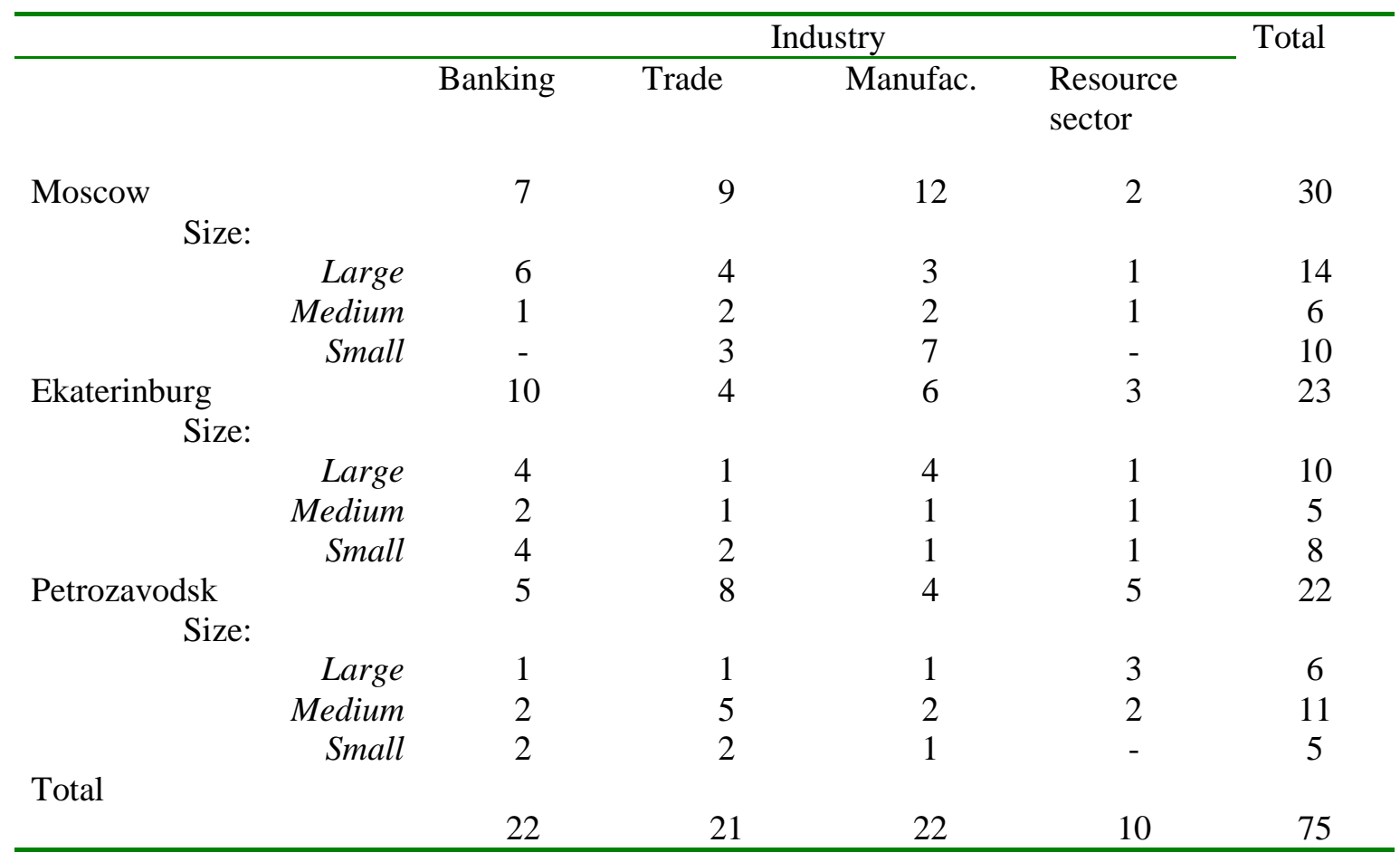

TABLE 2

Follow-up Interviews in 1999

\begin{tabular}{llc}
\hline & "Location" after 4 years & \\
\hline No-Contact: & Murdered & 9 \\
& Committed suicide & 2 \\
& Left the country & 1 \\
& Hiding from criminal charges & 2 \\
& Unreachable & 2 \\
& & \\
Not in Business: & Hired middle managers & 7 \\
& Civil servant & 2 \\
& Local politician & 1 \\
& Unemployed & 1 \\
& Retired & 1 \\
& & \\
& & 59 \\
In Business: & Refusal & 3 \\
& Interviewed & 56 \\
\hline
\end{tabular}


TABLE 3

Means, Standard Deviations and Pearson's Correlations

\begin{tabular}{|c|c|c|c|c|c|c|c|c|c|c|c|c|}
\hline & Variables & $\mathrm{M}$ & SD & 1 & 2 & 3 & 4 & 5 & 6 & 7 & 8 & 9 \\
\hline 1 & Network size & 34.4 & 9.42 & & & & & & & & & \\
\hline 2 & $\begin{array}{l}\text { Network } \\
\text { heterophily }\end{array}$ & 0.82 & 0.06 & .06 & & & & & & & & \\
\hline 3 & Strong ties & 12 & 4.70 & .781 & .04 & & & & & & & \\
\hline 4 & Weak ties & 22 & 6.44 & .891 & .05 & $.41 \Phi$ & & & & & & \\
\hline 5 & $\begin{array}{l}\text { Resourcefuln } \\
\text { ess }\end{array}$ & 16 & 6.6 & $.88 \mathrm{I}$ & .07 & $.61 \Phi$ & $.84 \pi$ & & & & & \\
\hline 6 & $\begin{array}{l}\text { Resources } \\
\text { mobilized } 95\end{array}$ & 0.41 & 0.49 & .18 & .15 & .10 & .19 & .17 & & & & \\
\hline 7 & $\begin{array}{l}\text { Sales growth } \\
96-95(\%)\end{array}$ & 0.19 & 0.58 & .08 & -.07 & .08 & .05 & .01 & .17 & & & \\
\hline 8 & $\begin{array}{l}\text { Sales growth } \\
97-96(\%)\end{array}$ & 0.04 & 0.37 & -.06 & .08 & -.16 & .02 & -.09 & .17 & $.32 *$ & & \\
\hline 9 & $\begin{array}{l}\text { Sales growth } \\
98-97(\%)\end{array}$ & -0.19 & 0.43 & .20 & -.12 & .00 & $.29 *$ & $.27 *$ & $.35^{*}$ & .03 & $.48 \mathrm{I}$ & \\
\hline 10 & $\begin{array}{l}\text { Average } \\
\text { sales growth } \\
(\%)\end{array}$ & 0.01 & 0.33 & .11 & -.06 & -.00 & .16 & .09 & $.32 *$ & $.72 \Phi$ & $.78 \mathbb{1}$ & .64 II \\
\hline 11 & $\begin{array}{l}\text { Profit margin } \\
95(\%)\end{array}$ & -0.32 & 2.66 & -.04 & -.05 & -.15 & .05 & -.03 & -.13 & -.06 & $.27 *$ & .27 \\
\hline 12 & $\begin{array}{l}\text { Profit margin } \\
96(\%)\end{array}$ & -0.32 & 1.85 & -.05 & .01 & -.13 & .01 & -.09 & -.17 & .16 & .23 & .01 \\
\hline 13 & $\begin{array}{l}\text { Profit margin } \\
97(\%)\end{array}$ & -0.71 & 3.23 & -.10 & -.10 & -.19 & -.02 & -.14 & -.23 & .06 & $.32 *$ & .15 \\
\hline 14 & $\begin{array}{l}\text { Profit margin } \\
98(\%)\end{array}$ & -0.46 & 1.16 & .27 & .26 & .09 & $.33^{*}$ & .20 & .03 & -.06 & .20 & $.30 *$ \\
\hline 15 & $\begin{array}{l}\text { Average } \\
\text { profit margin } \\
(\%)\end{array}$ & -0.22 & 0.86 & .04 & .15 & -.02 & .07 & -.08 & -.10 & .22 & .20 & -.05 \\
\hline 16 & $\begin{array}{l}\text { Return on } \\
\text { assets } 95(\%)\end{array}$ & 0.13 & 0.83 & -.04 & .25 & -.00 & -.06 & -.06 & .17 & -.03 & .13 & .25 \\
\hline 17 & $\begin{array}{l}\text { Return on } \\
\text { assets } 96(\%)\end{array}$ & 0.02 & 1.01 & .12 & .13 & .07 & .13 & .06 & .21 & .15 & .17 & .24 \\
\hline 18 & $\begin{array}{l}\text { Return on } \\
\text { assets } 97(\%)\end{array}$ & -0.13 & 0.99 & .16 & .08 & .06 & .18 & .07 & .18 & .19 & $.34 *$ & .39 II \\
\hline 19 & $\begin{array}{l}\text { Return on } \\
\text { assets } 98(\%)\end{array}$ & -0.40 & 1.40 & $.29 *$ & -.01 & .17 & $.29 *$ & .20 & .21 & .18 & .20 & $.38 \mathbb{I}$ \\
\hline 20 & $\begin{array}{l}\text { Average } \\
\text { return on } \\
\text { assets }(\%)\end{array}$ & -0.09 & 0.96 & .17 & .10 & .09 & .17 & .09 & .21 & .14 & .23 & .35 II \\
\hline
\end{tabular}


Table 3 (Con't)

\begin{tabular}{|c|c|c|c|c|c|c|c|c|c|c|c|}
\hline & Variables & 10 & 11 & 12 & 13 & 14 & 15 & 16 & 17 & 18 & 19 \\
\hline 11 & $\begin{array}{l}\text { Profit margin } \\
95(\%)\end{array}$ & .18 & & & & & & & & & \\
\hline 12 & $\begin{array}{l}\text { Profit margin } \\
96(\%)\end{array}$ & .19 & $.81 \Phi$ & & & & & & & & \\
\hline 13 & $\begin{array}{l}\text { Profit margin } \\
97(\%)\end{array}$ & .22 & .83 II & .879 & & & & & & & \\
\hline 14 & $\begin{array}{l}\text { Profit margin } \\
98(\%)\end{array}$ & .16 & .18 & .36 II & .26 & & & & & & \\
\hline 15 & $\begin{array}{l}\text { Average } \\
\text { profit margin } \\
(\%)\end{array}$ & .19 & $.40 \mathbb{I}$ & $.82 \pi$ & .879 & $.62 \pi$ & & & & & \\
\hline 16 & $\begin{array}{l}\text { Return on } \\
\text { assets } 95(\%)\end{array}$ & .14 & .24 & .24 & .19 & $.32 *$ & $.28 *$ & & & & \\
\hline 17 & $\begin{array}{l}\text { Return on } \\
\text { assets } 96(\%)\end{array}$ & .26 & .21 & $.32 *$ & .22 & .46 II & $.41 \Phi$ & $.82 \mathbb{I}$ & & & \\
\hline 18 & $\begin{array}{l}\text { Return on } \\
\text { assets } 97(\%)\end{array}$ & $.41 \Phi$ & .14 & .24 & .25 & $.47 \mathbb{I}$ & $.47 \mathbb{I}$ & $.75 \mathbb{I}$ & .879 & & \\
\hline 19 & $\begin{array}{l}\text { Return on } \\
\text { assets } 98(\%)\end{array}$ & $.35^{*}$ & .00 & .10 & .03 & $.50 \mathrm{I}$ & $.32 *$ & $.59 \mathrm{I}$ & .79 II & .90 II & \\
\hline 20 & $\begin{array}{l}\text { Average } \\
\text { return on } \\
\text { assets }(\%)\end{array}$ & $.33 *$ & .14 & .23 & .17 & $.49 \pi$ & $.40 \mathbb{I}$ & $.83 \Phi$ & .94 II & $.96 I$ & .929 \\
\hline
\end{tabular}


TABLE 4

Regression Analysis Predicting Firm Performance as Function of Initial Networks (N=56)

\begin{tabular}{|c|c|c|c|c|c|c|c|}
\hline & \multicolumn{4}{|c|}{ Sales growth in 1998} & \multirow{2}{*}{ 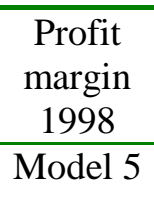 } & \multirow{2}{*}{$\begin{array}{c}\text { Return } \\
\text { on assets } \\
1998 \\
\text { Model } 6\end{array}$} & \multirow{2}{*}{$\begin{array}{c}\begin{array}{c}\text { Average } \\
\text { sales } \\
\text { growth }\end{array} \\
\text { Model } 7\end{array}$} \\
\hline & Model 1 & Model 2 & Model 3 & Model 4 & & & \\
\hline $\begin{array}{l}\text { Industry } \\
\text { Dummies }\end{array}$ & & & & & & & \\
\hline Banking & $\begin{array}{l}-0.14 \\
(0.23)\end{array}$ & $\begin{array}{l}-0.22 \\
(0.22)\end{array}$ & $\begin{array}{l}-0.07 \\
(0.20)\end{array}$ & $\begin{array}{l}-0.27 \\
(0.20)\end{array}$ & $\begin{array}{l}-0.56 \\
(0.58)\end{array}$ & $\begin{array}{l}-0.12 \\
(0.70)\end{array}$ & $\begin{array}{l}-0.15 \\
(0.15)\end{array}$ \\
\hline Trade & $\begin{array}{l}-0.15 \\
(0.20)\end{array}$ & $\begin{array}{l}-0.28 \\
(0.21)\end{array}$ & $\begin{array}{l}-0.17 \\
(0.19)\end{array}$ & $\begin{array}{l}-0.41 \\
(0.17)\end{array}$ & $\begin{array}{l}-0.14 \\
(0.56)\end{array}$ & $\begin{array}{l}-0.18 \\
(0.67)\end{array}$ & $\begin{array}{l}-0.16 \\
(0.14)\end{array}$ \\
\hline Manufacturing & $\begin{array}{l}-0.03 \\
(0.21)\end{array}$ & $\begin{array}{l}-0.18 \\
(0.21)\end{array}$ & $\begin{array}{c}0.08 \\
(0.19)\end{array}$ & $\begin{array}{l}-0.16 \\
(0.18)\end{array}$ & $\begin{array}{l}-0.17 \\
(0.56)\end{array}$ & $\begin{array}{l}-0.34 \\
(0.68)\end{array}$ & $\begin{array}{c}0.03 \\
(0.14)\end{array}$ \\
\hline Size dummies & & & & & & & \\
\hline Small & $\begin{array}{l}-0.03 \\
(0.14)\end{array}$ & $\begin{array}{c}0.09 \\
(0.15)\end{array}$ & $\begin{array}{l}-0.02 \\
(0.14)\end{array}$ & & $\begin{array}{l}-0.65 \\
(0.40)\end{array}$ & $\begin{array}{l}-0.64 \\
(0.47)\end{array}$ & $\begin{array}{c}0.11 \\
(0.10)\end{array}$ \\
\hline Large & $\begin{array}{c}0.29 \\
(0.14)\end{array}$ & $\begin{array}{c}0.20 \\
(0.14)\end{array}$ & $\begin{array}{c}0.17 \\
(0.14)\end{array}$ & & $\begin{array}{l}-0.12 \\
(0.38)\end{array}$ & $\begin{array}{c}0.01 \\
(0.46)\end{array}$ & $\begin{array}{c}0.08 \\
(0.11)\end{array}$ \\
\hline $\begin{array}{l}\text { New venture } \\
\text { dummy } \\
\text { Region } \\
\text { dummies }\end{array}$ & $\begin{array}{c}0.22 \\
(0.13)\end{array}$ & $\begin{array}{c}0.22 \\
(0.12)\end{array}$ & $\begin{array}{c}0.23 \\
(0.12)\end{array}$ & $\begin{array}{c}0.26 \\
(0.11)\end{array}$ & $\begin{array}{c}0.46 \\
(0.33)\end{array}$ & $\begin{array}{c}0.37 \\
(0.39)\end{array}$ & $\begin{array}{c}0.19 \\
(0.09)\end{array}$ \\
\hline Moscow & $\begin{array}{l}-0.09 \\
(0.15)\end{array}$ & & & $\begin{array}{l}-0.01 \\
(0.14)\end{array}$ & & & \\
\hline Ekaterinburg & $\begin{array}{c}0.30 \\
(0.17)\end{array}$ & & & $\begin{array}{c}0.31 \\
(0.15)\end{array}$ & & & \\
\hline $\begin{array}{l}\text { Independent } \\
\text { variables }\end{array}$ & & & & & & & \\
\hline Weak ties & & $\begin{array}{l}0.02 * \\
(0.01)\end{array}$ & & $\begin{array}{l}0.01^{*} \\
(0.00)\end{array}$ & $\begin{array}{l}0.05^{*} \\
(0.02)\end{array}$ & $\begin{array}{l}0.06^{*} \\
(0.03)\end{array}$ & \\
\hline $\begin{array}{l}\text { Resources } \\
\text { mobilized } 95\end{array}$ & & & $\begin{array}{l}0.35 \mathbb{I} \\
(0.12)\end{array}$ & $\begin{array}{l}0.37 \Phi \\
(0.11)\end{array}$ & & & $\begin{array}{l}0.26 \mathbb{I} \\
(0.09)\end{array}$ \\
\hline Model $F$ & 1.65 & 1.61 & $2.11 *$ & $3.50 \mathbb{1}$ & 1.84 & 1.90 & $1.97 *$ \\
\hline$R^{2}$ & 0.23 & 0.20 & 0.25 & 0.39 & 0.23 & 0.23 & 0.17 \\
\hline
\end{tabular}

Values represent unstandardized coefficients; standard errors are in parentheses.

$* p<.05$

II $p<.01$ 


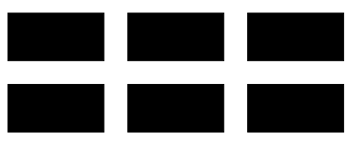

THE WILLIAM DAVIDSON INSTITUTE

AT THE UNIVERSITY OF MICHIGAN BUSINESSSCHOOL

\section{DAVIDSON INSTITUTE WORKING PAPER SERIES}

Working Papers are available at: www.wdi.bus.umich.edu

CURRENT AS OF $12 / 20 / 00$

\begin{tabular}{|c|c|c|}
\hline Publication & Authors & Date \\
\hline $\begin{array}{l}\text { No. } 352 \text { Social Capital and Entrepreneurial Performance in Russia: A } \\
\text { Panel Study }\end{array}$ & Bat Batjargal & Dec. 2000 \\
\hline $\begin{array}{l}\text { No. 351 Entrepreneurial Versatility, Resources and Firm Performance in } \\
\text { Russia: A Panel Study }\end{array}$ & Bat Batjargal & Dec. 2000 \\
\hline $\begin{array}{l}\text { No. } 350 \text { The Dynamics of Entrepreneurial Networks in a Transitional } \\
\text { Economy: The Case of Russia }\end{array}$ & Bat Batjargal & Dec. 2000 \\
\hline $\begin{array}{l}\text { No. } 349 \text { R\&D and Technology Spillovers via FDI: Innovation and } \\
\text { Absorptive Capacity }\end{array}$ & Yuko Kinoshita & Nov. 2000 \\
\hline $\begin{array}{l}\text { No. } 348 \text { Microeconomic aspects of Economic Growth in Eastern } \\
\text { Europe and the Former Soviet Union, } 1950-2000\end{array}$ & Sergei Guriev and Barry W. Ickes & Nov. 2000 \\
\hline $\begin{array}{l}\text { No. } 347 \text { Effective versus Statutory Taxation: Measuring Effective Tax } \\
\text { Administration in Transition Economies }\end{array}$ & $\begin{array}{l}\text { Mark E. Schaffer and Gerard } \\
\text { Turley }\end{array}$ & Nov. 2000 \\
\hline $\begin{array}{l}\text { No. } 346 \text { Objectives and Constraints of Entrepreneurs: Evidence from } \\
\text { Small and Medium Size Enterprises in Russia and Bulgaria }\end{array}$ & $\begin{array}{l}\text { Francesca Pissarides, Miroslav } \\
\text { Singer and Jan Svejnar }\end{array}$ & Oct. 2000 \\
\hline No. 345 Corruption and Anticorruption in the Czech Republic & $\begin{array}{l}\text { Lubomír Lízal and Evžen } \\
\text { Kočenda }\end{array}$ & Oct. 2000 \\
\hline No. 344 The Effects of Direct Foreign Investment on Domestic Firms & Jozef Konings & Oct. 2000 \\
\hline No. 343 On the Identification of Relative Wage Rigidity Dynamics & ck A. Puhani & Oct. 2000 \\
\hline $\begin{array}{l}\text { No. } 342 \text { The Determinants of Foreign Direct Investment in Transition } \\
\text { Economies }\end{array}$ & Alan A. Bevan and Saul Estrin & Oct. 2000 \\
\hline No. 341 The Global Spread of Stock Exchanges, 1980-1998 & Klaus Weber and Gerald F. Davis & Nov. 2000 \\
\hline $\begin{array}{l}\text { No. } 340 \text { The Costs and Benefits of Euro-isation in Central-Eastern } \\
\text { Europe Before or Instead of EMU Membership }\end{array}$ & D. Mario Nuti & Oct. 2000 \\
\hline No. 339 Debt Overhang and Barter in Russia & $\begin{array}{l}\text { Sergei Guriev, Igor Makarov and } \\
\text { Mathilde Maurel }\end{array}$ & Sept. 2000 \\
\hline $\begin{array}{l}\text { No. } 338 \text { Firm Performance and the Political Economy of Corporate } \\
\text { Governance: Survey Evidence for Bulgaria, Hungary, Slovakia and } \\
\text { Slovenia }\end{array}$ & $\begin{array}{l}\text { Patrick Paul Walsh and Ciara } \\
\text { Whela }\end{array}$ & July 2000 \\
\hline No. 337 Investment and Instability & $\begin{array}{l}\text { Nauro F. Campos and Jeffrey B. } \\
\text { Nugent }\end{array}$ & May 2000 \\
\hline $\begin{array}{l}\text { No. } 336 \text { The Evolution of the Insurance Sector in Central and } \\
\text { Eastern Europe and the former Soviet Union }\end{array}$ & Robert B.K. Pye & Aug. 2000 \\
\hline $\begin{array}{l}\text { No. } 335 \text { Institutional Technology and the Chains of Trust: Capital } \\
\text { Markets and Privatization in Russia and the Czech Republic }\end{array}$ & Bruce Kogut and Andrew Spicer & Aug. 2000 \\
\hline No. 334 The Evolution of Market Integration in Russia & $\begin{array}{l}\text { Daniel Berkowitz and David N. } \\
\text { DeJong }\end{array}$ & Aug. 2000 \\
\hline No. 333 Efficiency and Market Share in Hungarian Corporate Sector & László Halpern and Gábor Körösi & July 2000 \\
\hline No. 332 Search-Money-and-Barter Models of Financial Stabilization & $\begin{array}{l}\text { S.I. Boyarchenko and S.Z. } \\
\text { Levendorskii }\end{array}$ & July 2000 \\
\hline $\begin{array}{l}\text { No. } 331 \text { Worker Training in a Restructuring Economy: Evidence from } \\
\text { the Russian Transition }\end{array}$ & $\begin{array}{l}\text { Mark C. Berger, John S. Earle } \\
\text { and Klara Z. Sabirianova }\end{array}$ & Aug. 2000 \\
\hline $\begin{array}{l}\text { No. } 330 \text { Economic Development in Palanpur 1957-1993: A Sort of } \\
\text { Growth }\end{array}$ & Peter Lanjouw & Aug. 2000 \\
\hline $\begin{array}{l}\text { No. } 329 \text { Trust, Organizational Controls, Knowledge Acquisition from } \\
\text { the Foreign Parents, and Performance in Vietnamese International Joint } \\
\text { Ventures }\end{array}$ & $\begin{array}{l}\text { Marjorie A. Lyles, Le Dang } \\
\text { Doanh, and Jeffrey Q. Barden }\end{array}$ & June 2000 \\
\hline
\end{tabular}




\begin{tabular}{|c|c|c|}
\hline $\begin{array}{l}\text { No. } 328 \text { Comparative Advertising in the Global Marketplace: The } \\
\text { Effects of Cultural Orientation on Communication }\end{array}$ & $\begin{array}{l}\text { Zeynep Gürhan-Canli and } \\
\text { Durairaj Maheswaran }\end{array}$ & Aug. 2000 \\
\hline No. 327 Post Privatization Enterprise Restructuring & Morris Bornstein & July 2000 \\
\hline No. 326 Who is Afraid of Political Instability? & $\begin{array}{l}\text { Nauro F. Campos and Jeffrey B. } \\
\text { Nugent }\end{array}$ & July 2000 \\
\hline No. 325 Business Groups, the Financial Market and Modernization & Raja Kali & June 2000 \\
\hline $\begin{array}{l}\text { No. } 324 \text { Restructuring with What Success? A Case Study of Russian } \\
\text { Firms }\end{array}$ & Susan Linz & July 2000 \\
\hline $\begin{array}{l}\text { No. } 323 \text { Priorities and Sequencing in Privatization: Theory and } \\
\text { Evidence from the Czech Republic }\end{array}$ & $\begin{array}{l}\text { Nandini Gupta, John C. Ham and } \\
\text { Jan Svejnar }\end{array}$ & May 2000 \\
\hline $\begin{array}{l}\text { No. } 322 \text { Liquidity, Volatility, and Equity Trading Costs Across } \\
\text { Countries and Over Time }\end{array}$ & $\begin{array}{l}\text { Ian Domowitz, Jack Glen and } \\
\text { Ananth Madhavan }\end{array}$ & Mar. 2000 \\
\hline $\begin{array}{l}\text { No. } 321 \text { Equilibrium Wage Arrears: A Theoretical and Empirical } \\
\text { Analysis of Institutional Lock-In }\end{array}$ & $\begin{array}{l}\text { John S. Earle and Klara Z. } \\
\text { Sabirianova }\end{array}$ & Oct. 2000 \\
\hline No. 320 Rethinking Marketing Programs for Emerging Markets & $\begin{array}{l}\text { Niraj Dawar and Amitava } \\
\text { Chattopadhyay }\end{array}$ & June 2000 \\
\hline $\begin{array}{l}\text { No. } 319 \text { Public Finance and Low Equilibria in Transition Economies: } \\
\text { the Role of Institutions }\end{array}$ & $\begin{array}{l}\text { Daniel Daianu and Radu } \\
\text { Vranceanu }\end{array}$ & June 2000 \\
\hline $\begin{array}{l}\text { No. } 318 \text { Some Econometric Evidence on the Effectiveness of Active } \\
\text { Labour Market Programmes in East Germany }\end{array}$ & $\begin{array}{l}\text { Martin Eichler and Michael } \\
\text { Lechner }\end{array}$ & June 2000 \\
\hline No. 317 A Model of Russia's "Virtual Economy" & R.E Ericson and B.W Ickes & May 2000 \\
\hline $\begin{array}{l}\text { No. } 316 \text { Financial Institutions, Financial Contagion, and Financial } \\
\text { Crises }\end{array}$ & $\begin{array}{l}\text { Haizhou Huang and Chenggang } \\
\mathrm{Xu}\end{array}$ & Mar. 2000 \\
\hline $\begin{array}{l}\text { No. } 315 \text { Privatization versus Regulation in Developing Economies: The } \\
\text { Case of West African Banks }\end{array}$ & $\begin{array}{l}\text { Jean Paul Azam, Bruno Biais, and } \\
\text { Magueye Dia }\end{array}$ & Feb. 2000 \\
\hline $\begin{array}{l}\text { No. } 314 \text { Is Life More Risky in the Open? Household Risk-Coping and } \\
\text { the Opening of China's Labor Markets }\end{array}$ & John Giles & Apr. 2000 \\
\hline $\begin{array}{l}\text { No. } 313 \text { Networks, Migration and Investment: Insiders and Outsiders in } \\
\text { Tirupur's Production Cluster }\end{array}$ & $\begin{array}{l}\text { Abhijit Banerjee and Kaivan } \\
\text { Munshi }\end{array}$ & Mar. 2000 \\
\hline $\begin{array}{l}\text { No. } 312 \text { Computational Analysis of the Impact on India of the Uruguay } \\
\text { Round and the Forthcoming WTO Trade Negotiations }\end{array}$ & $\begin{array}{l}\text { Rajesh Chadha, Drusilla K. } \\
\text { Brown, Alan V. Deardorff and } \\
\text { Robert M. Stern }\end{array}$ & Mar. 2000 \\
\hline No. 311 Subsidized Jobs for Unemployed Workers in Slovakia & Jan. C. van Ours & May 2000 \\
\hline No. 310 Determinants of Managerial Pay in the Czech Republic & $\begin{array}{l}\text { Tor Eriksson, Jaromir Gottvald } \\
\text { and Pavel Mrazek }\end{array}$ & May 2000 \\
\hline $\begin{array}{l}\text { No. } 309 \text { The Great Human Capital Reallocation: An Empirical Analysis } \\
\text { of Occupational Mobility in Transitional Russia }\end{array}$ & Klara Z. Sabirianova & Oct. 2000 \\
\hline No. 308 Economic Development, Legality, and the Transplant Effect & $\begin{array}{l}\text { Daniel Berkowitz, Katharina } \\
\text { Pistor, and Jean-Francois Richard }\end{array}$ & Feb. 2000 \\
\hline $\begin{array}{l}\text { No. } 307 \text { Community Participation, Teacher Effort, and Educational } \\
\text { Outcome: The Case of El Salvador's EDUCO Program }\end{array}$ & Yasuyuki Sawada & Nov. 1999 \\
\hline No. 306 Gender Wage Gap and Segregation in Late Transition & Stepan Jurajda & May 2000 \\
\hline $\begin{array}{l}\text { No. } 305 \text { The Gender Pay Gap in the Transition from Communism: } \\
\text { Some Empirical Evidence }\end{array}$ & Andrew Newell and Barry Reilly & May 2000 \\
\hline No. 304 Post-Unification Wage Growth in East Germany & Jennifer Hunt & Nov. 1998 \\
\hline $\begin{array}{l}\text { No. } 303 \text { How Does Privatization Affect Workers? The Case of the } \\
\text { Russian Mass Privatization Program }\end{array}$ & Elizabeth Brainerd & May 2000 \\
\hline $\begin{array}{l}\text { No. } 302 \text { Liability for Past Environmental Contamination and } \\
\text { Privatization }\end{array}$ & Dietrich Earnhart & Mar. 2000 \\
\hline No. 301 Varieties, Jobs and EU Enlargement & $\begin{array}{l}\text { Tito Boeri and Joaquim Oliveira } \\
\text { Martins }\end{array}$ & May 2000 \\
\hline No. 300 Employer Size Effects in Russia & Todd Idson & Apr. 2000 \\
\hline $\begin{array}{l}\text { No. } 299 \text { Information Complements, Substitutes, and Strategic Product } \\
\text { Design }\end{array}$ & $\begin{array}{l}\text { Geoffrey G. Parker and Marshall } \\
\text { W. Van Alstyne }\end{array}$ & Mar. 2000 \\
\hline $\begin{array}{l}\text { No. } 298 \text { Markets, Human Capital, and Inequality: Evidence from Rural } \\
\text { China }\end{array}$ & $\begin{array}{l}\text { Dwayne Benjamin, Loren Brandt, } \\
\text { Paul Glewwe, and Li Guo }\end{array}$ & May 2000 \\
\hline
\end{tabular}


Davidson Institute Working Papers are available at: www.wdi.bus.umich.edu

\begin{tabular}{|c|c|c|}
\hline No. 297 Corporate Governance in the Asian Financial Crisis & $\begin{array}{l}\text { Simon Johnson, Peter Boone, } \\
\text { Alasdair Breach, and Eric } \\
\text { Friedman }\end{array}$ & Nov. 1999 \\
\hline No. 296 Competition and Firm Performance: Lessons from Russia & J. David Brown and John S. Earle & Mar. 2000 \\
\hline No. 295 Wage Determination in Russia: An Econometric Investigation & $\begin{array}{l}\text { Peter J. Luke and Mark E. } \\
\text { Schaffer }\end{array}$ & Mar. 2000 \\
\hline $\begin{array}{l}\text { No. 294: Can Banks Promote Enterprise Restructuring?: Evidence From } \\
\text { a Polish Bank's Experience }\end{array}$ & John P. Bonin and Bozena Leven & Mar. 2000 \\
\hline No. 293: Why do Governments Sell Privatised Companies Abroad? & $\begin{array}{l}\text { Bernardo Bortolotti, Marcella } \\
\text { Fantini and Carlo Scarpa }\end{array}$ & Mar. 2000 \\
\hline $\begin{array}{l}\text { No. 292: Going Public in Poland: Case-by-Case Privatizations, Mass } \\
\text { Privatization and Private Sector Initial Public Offerings }\end{array}$ & Wolfgang Aussenegg & Dec. 1999 \\
\hline $\begin{array}{l}\text { No. 291: Institutional Technology and the Chains of Trust: Capital } \\
\text { Markets and Privatization in Russia and the Czech Republic }\end{array}$ & Bruce Kogut and Andrew Spicer & Mar. 1999 \\
\hline No. 290: Banking Crises and Bank Rescues: The Effect of Reputation & Jenny Corbett and Janet Mitchell & Jan. 2000 \\
\hline $\begin{array}{l}\text { No. 289: Do Active Labor Market Policies Help Unemployed Workers } \\
\text { to Find and Keep Regular Jobs? }\end{array}$ & Jan C. van Ours & Feb. 2000 \\
\hline No. 288: Consumption Patterns of the New Elite in Zimbabwe & Russell Belk & Feb. 2000 \\
\hline $\begin{array}{l}\text { No. 287: Barter in Transition Economies: Competing Explanations } \\
\text { Confront Ukranian Data }\end{array}$ & $\begin{array}{l}\text { Dalia Marin, Daniel Kaufmann } \\
\text { and Bogdan Gorochowskij }\end{array}$ & Jan. 2000 \\
\hline $\begin{array}{l}\text { No. 286: The Quest for Pension Reform: Poland's Security through } \\
\text { Diversity }\end{array}$ & $\begin{array}{l}\text { Marek Góra and Michael } \\
\text { Rutkowski }\end{array}$ & Jan. 2000 \\
\hline No. 285: Disorganization and Financial Collapse & $\begin{array}{l}\text { Dalia Marin and Monika } \\
\text { Schnitzer }\end{array}$ & Oct. 1999 \\
\hline No. 284: Coordinating Changes in M-form and U-form Organizations & $\begin{array}{l}\text { Yingyi Qian, Gérard Roland and } \\
\text { Chenggang Xu }\end{array}$ & May 1999 \\
\hline $\begin{array}{l}\text { No. 283: Why Russian Workers Do Not Move: Attachment of Workers } \\
\text { Through In-Kind Payments }\end{array}$ & Guido Friebel and Sergei Guriev & Oct. 1999 \\
\hline No. 282: Lessons From Fiascos in Russian Corporate Governance & $\begin{array}{l}\text { Merritt B. Fox and Michael A. } \\
\text { Heller }\end{array}$ & Oct. 1999 \\
\hline $\begin{array}{l}\text { No. 281: Income Distribution and Price Controls: Targeting a Social } \\
\text { Safety Net During Economic Transition }\end{array}$ & $\begin{array}{l}\text { Michael Alexeev and James } \\
\text { Leitzel }\end{array}$ & Mar. 1999 \\
\hline $\begin{array}{l}\text { No. 280: Starting Positions, Reform Speed, and Economic Outcomes in } \\
\text { Transitioning Economies }\end{array}$ & William Hallagan and Zhang Jun & Jan. 2000 \\
\hline No. 279 : The Value of Prominent Directors & $\begin{array}{l}\text { Yoshiro Miwa \& J. Mark } \\
\text { Ramseyer }\end{array}$ & Oct. 1999 \\
\hline No. 278: The System Paradigm & János Kornai & Apr. 1998 \\
\hline $\begin{array}{l}\text { No. 277: The Developmental Consequences of Foreign Direct } \\
\text { Investment in the Transition from Socialism to Capitalism: The } \\
\text { Performance of Foreign Owned Firms in Hungary }\end{array}$ & Lawrence Peter King & Sept. 1999 \\
\hline $\begin{array}{l}\text { No. 276: Stability and Disorder: An Evolutionary Analysis of Russia's } \\
\text { Virtual Economy }\end{array}$ & $\begin{array}{l}\text { Clifford Gaddy and Barry W. } \\
\text { Ickes }\end{array}$ & Nov. 1999 \\
\hline $\begin{array}{l}\text { No. 275: Limiting Government Predation Through Anonymous } \\
\text { Banking: A Theory with Evidence from China. }\end{array}$ & $\begin{array}{l}\text { Chong-En Bai, David D. Li, } \\
\text { Yingyi Qian and Yijiang Wang }\end{array}$ & July 1999 \\
\hline No. 274: Transition with Labour Supply & Tito Boeri & Dec. 1999 \\
\hline $\begin{array}{l}\text { No. 273: Sectoral Restructuring and Labor Mobility: A Comparative } \\
\text { Look at the Czech Republic }\end{array}$ & Vit Sorm and Katherine Terrell & Nov. 1999 \\
\hline $\begin{array}{l}\text { No. 272: Published in: Journal of Comparative Economics "Returns to } \\
\text { Human Capital Under the Communist Wage Grid and During the } \\
\text { Transition to a Market Economy" Vol. 27, pp. 33-60 } 1999 .\end{array}$ & $\begin{array}{l}\text { Daniel Munich, Jan Svejnar and } \\
\text { Katherine Terrell }\end{array}$ & Oct. 1999 \\
\hline $\begin{array}{l}\text { No. 271: Barter in Russia: Liquidity Shortage Versus Lack of } \\
\text { Restructuring }\end{array}$ & $\begin{array}{l}\text { Sophie Brana and Mathilde } \\
\text { Maurel }\end{array}$ & June 1999 \\
\hline $\begin{array}{l}\text { No. 270: Tests for Efficient Financial Intermediation with Application to } \\
\text { China }\end{array}$ & Albert Park and Kaja Sehrt & Mar. 1999 \\
\hline $\begin{array}{l}\text { No. 269a: Russian Privatization and Corporate Governance: What Went } \\
\text { Wrong? }\end{array}$ & $\begin{array}{l}\text { Bernard Black, Reinier Kraakman } \\
\text { and Anna Tarassova }\end{array}$ & May 2000 \\
\hline
\end{tabular}




\begin{tabular}{|c|c|c|}
\hline $\begin{array}{l}\text { No. 269: Russian Privatization and Corporate Governance: What Went } \\
\text { Wrong? }\end{array}$ & $\begin{array}{l}\text { Bernard Black, Reinier Kraakman } \\
\text { and Anna Tarassova }\end{array}$ & Sept. 1999 \\
\hline No. 268: Are Russians Really Ready for Capitalism? & Susan Linz & Sept. 1999 \\
\hline No. 267: Do Stock Markets Promote Economic Growth? & $\begin{array}{l}\text { Randall K. Filer, Jan Hanousek } \\
\text { and Nauro Campos }\end{array}$ & Sept. 1999 \\
\hline $\begin{array}{l}\text { No. 266: Objectivity, Proximity and Adaptability in Corporate } \\
\text { Governance }\end{array}$ & $\begin{array}{l}\text { Arnoud W.A Boot and Jonathan } \\
\text { R. Macey }\end{array}$ & Sept. 1999 \\
\hline $\begin{array}{l}\text { No. 265: When the Future is not What it Used to Be: Lessons from the } \\
\text { Western European Experience to Forecasting Education and Training in } \\
\text { Transitional Economies }\end{array}$ & $\begin{array}{l}\text { Nauro F. Campos, Gerard } \\
\text { Hughes, Stepan Jurajda, and } \\
\text { Daniel Munich }\end{array}$ & Sept. 1999 \\
\hline $\begin{array}{l}\text { No. 264: The Institutional Foundation of Foreign-Invested Enterprises } \\
\text { (FIEs) in China }\end{array}$ & Yasheng Huang & Sept. 1999 \\
\hline $\begin{array}{l}\text { No. 263: The Changing Corporate Governance Paradigm: Implications } \\
\text { for Transition and Developing Countries }\end{array}$ & $\begin{array}{l}\text { Erik Berglof and Ernst-Ludwig } \\
\text { von Thadden }\end{array}$ & June 1999 \\
\hline No. 262: Law Enforcement and Transition & $\begin{array}{l}\text { Gerard Roland and Thierry } \\
\text { Verdier }\end{array}$ & May 1999 \\
\hline $\begin{array}{l}\text { No. 261: Soft Budget Constraints, Pecuniary Externality, and the Dual } \\
\text { Track System }\end{array}$ & Jiahua Che & June 2000 \\
\hline $\begin{array}{l}\text { No. 260: Missing Market in Labor Quality: The Role of Quality Markets } \\
\text { in Transition }\end{array}$ & Gary H. Jefferson & July 1999 \\
\hline $\begin{array}{l}\text { No. 259: Do Corporate Global Environmental Standards in Emerging } \\
\text { Markets Create or Destroy Market Value }\end{array}$ & $\begin{array}{l}\text { Glen Dowell, Stuart Hart and } \\
\text { Bernard Yeung }\end{array}$ & June 1999 \\
\hline No. 258: Public Training and Outflows from Unemployment & Patrick A. Puhani & June 1999 \\
\hline $\begin{array}{l}\text { No. 257: Ownership Versus Environment: Why are Public Sector Firms } \\
\text { Inefficient? }\end{array}$ & $\begin{array}{l}\text { Ann P. Bartel and Ann E. } \\
\text { Harrison }\end{array}$ & June 1999 \\
\hline $\begin{array}{l}\text { No. 256: Taxation and Evasion in the Presence of Exortion by } \\
\text { Organized Crime }\end{array}$ & $\begin{array}{l}\text { Michael Alexeev, Eckhard Janeba } \\
\text { and Stefan Osborne }\end{array}$ & Nov. 1999 \\
\hline No. 255: Revisiting Hungary’s Bankruptcy Episode & $\begin{array}{l}\text { John P. Bonin and Mark E. } \\
\text { Schaffer }\end{array}$ & Sept. 1999 \\
\hline No. 254: FDI in Emerging Markets: A Home-Country View & Marina v.N Whitman & June 1999 \\
\hline $\begin{array}{l}\text { No. 253: The Asian Financial Crisis: What Happened, and What is to be } \\
\text { Done }\end{array}$ & $\begin{array}{l}\text { Jeffrey D. Sachs and Wing Thye } \\
\text { Woo }\end{array}$ & Jan. 1999 \\
\hline No. 252: Organizational Law as Asset Partitioning & $\begin{array}{l}\text { Henry Hansmann and Reinier } \\
\text { Kraakman }\end{array}$ & Sept. 1999 \\
\hline $\begin{array}{l}\text { No. 251: Consumer Behavior Research in Emerging Consumer Markets: } \\
\text { the Case of the Optimum Stimulation Level in South Africa }\end{array}$ & $\begin{array}{l}\text { Jan-Benedict E. M. Steenkamp } \\
\text { and Steven M. Burgess }\end{array}$ & Sept. 1999 \\
\hline $\begin{array}{l}\text { No. 250: Property Rights Formation and the Organization of Exchange } \\
\text { and Production in Rural China }\end{array}$ & $\begin{array}{l}\text { Matthew A. Turner, Loren } \\
\text { Brandt, and Scott Rozelle }\end{array}$ & July 1998 \\
\hline $\begin{array}{l}\text { No. 249: Impacts of the Indonesian Economic Crisis: Price Changes and } \\
\text { the Poor }\end{array}$ & $\begin{array}{l}\text { James Levinsohn, Steven Berry, } \\
\text { and Jed Friedman }\end{array}$ & June 1999 \\
\hline $\begin{array}{l}\text { No. 248: Internal Barriers in the Transition of Enterprises from Central } \\
\text { Plan to Market }\end{array}$ & Charalambos Vlachoutsicos & July 1999 \\
\hline $\begin{array}{l}\text { No. 247: Spillovers from Multinationals in Developing Countries: the } \\
\text { Mechanisms at Work }\end{array}$ & Richard E. Caves & June 1999 \\
\hline $\begin{array}{l}\text { No. 246: Dynamism and Inertia on the Russian Labour Market: A } \\
\text { Model of Segmentation }\end{array}$ & $\begin{array}{l}\text { Irena Grosfeld, Claudia Senik- } \\
\text { Leygonie, Thierry Verdier, Stanislav } \\
\text { Kolenikov and Elena Paltseva }\end{array}$ & May 1999 \\
\hline No. 245: Lessons from Bank Privatization in Central Europe & John Bonin and Paul Wachtel & May 1999 \\
\hline $\begin{array}{l}\text { No. 244: Nominal-Real Tradeoffs and the Effects of Monetary Policy: } \\
\text { the Romanian Experience }\end{array}$ & Christian Popa & Dec. 1998 \\
\hline $\begin{array}{l}\text { No. 243: Privatization, Political Risk and Stock Market Development in } \\
\text { Emerging Economies }\end{array}$ & $\begin{array}{l}\text { Enrico C. Perotti and Pieter van } \\
\text { Oijen }\end{array}$ & Mar. 1999 \\
\hline No. 242: Investment Financing in Russian Financial-Industrial Groups & $\begin{array}{l}\text { Enrico C. Perotti and Stanislav } \\
\text { Gelfer }\end{array}$ & Oct. 1998 \\
\hline $\begin{array}{l}\text { No. 241: Can governments maintain hard budget constraints? Bank } \\
\text { lending and financial isolation in Romania }\end{array}$ & $\begin{array}{l}\text { Octavian Carare, Constantijn } \\
\text { Claessens, Enrico C. Perotti }\end{array}$ & Jan. 1999 \\
\hline
\end{tabular}




\begin{tabular}{|c|c|c|}
\hline $\begin{array}{l}\text { No. 240: Democratic Institutions and Economic Reform: the Polish } \\
\text { Case }\end{array}$ & $\begin{array}{l}\text { John E. Jackson, Jacek Klich, and } \\
\text { Krystyna Poznanska }\end{array}$ & Apr. 1998 \\
\hline No. 239: A Longitudinal Study of IJV Performance in Eastern Europe & $\begin{array}{l}\text { Keith D. Brouthers and Gary } \\
\text { Bamossy }\end{array}$ & June 1999 \\
\hline $\begin{array}{l}\text { No. 238: Published in: Journal of Business Venturing, "Firm Creation } \\
\text { and Economic Transitions" } 14(5,6) \text { Sep/Nov 1999, pp. 427-450. }\end{array}$ & $\begin{array}{l}\text { John E. Jackson, Jacek Klich, } \\
\text { Krystyna Poznanska }\end{array}$ & July 1998 \\
\hline No. 237: Analysis of Entrepreneurial Attitudes in Poland & $\begin{array}{l}\text { John E. Jackson and Aleksander } \\
\text { S. Marcinkowski }\end{array}$ & Mar. 1997 \\
\hline $\begin{array}{l}\text { No. 236: Investment and Finance in De Novo Private Firms: Empirical } \\
\text { Results from the Czech Republic, Hungary, and Poland }\end{array}$ & $\begin{array}{l}\text { Andrzej Bratkowski, Irena } \\
\text { Grosfeld, Jacek Rostowski }\end{array}$ & Apr. 1999 \\
\hline $\begin{array}{l}\text { No. 235: Does a Soft Macroeconomic Environment Induce } \\
\text { Restructuring on the Microeconomic Level during the Transition } \\
\text { Period? Evidence from Investment Behavior of Czech Enterprises }\end{array}$ & Lubomír Lízal & June 1999 \\
\hline $\begin{array}{l}\text { No. 234: Banking Reform in China: Gradually Strengthening Pillar or } \\
\text { Fragile Reed? }\end{array}$ & John Bonin & June 1999 \\
\hline $\begin{array}{l}\text { No. 233: Theories of Soft Budget Constraints and the Analysis of } \\
\text { Banking Crises }\end{array}$ & Janet Mitchell & Mar. 1999 \\
\hline $\begin{array}{l}\text { No. 232: Unemployment Risk, Precautionary Savings, and } \\
\text { Moonlighting in Russia }\end{array}$ & $\begin{array}{l}\text { Alessandra Guariglia and Byung- } \\
\text { Yeon Kim }\end{array}$ & June 1999 \\
\hline $\begin{array}{l}\text { No. 231: Investing in Turbulent Times: The Investment Behavior of } \\
\text { Polish Firms in the Transition }\end{array}$ & $\begin{array}{l}\text { Josef C. Brada, Arthur E. King, } \\
\text { and Chia-Ying Ma }\end{array}$ & Apr. 1999 \\
\hline No. 230: The End of Moderate Inflation in Three Transition Economies? & Josef C. Brada and Ali M. Kutan & Apr. 1999 \\
\hline $\begin{array}{l}\text { No. 229: Back to the Future: The Growth Prospects of Transition } \\
\text { Economies Reconsidered }\end{array}$ & Nauro F. Campos & Apr. 1999 \\
\hline No. 228: The Enterprise Isolation Program in Russia & Simeon Djankov & Apr. 1999 \\
\hline $\begin{array}{l}\text { No. 227: Published in: Journal of Comparative Economics, "Ownership } \\
\text { Concentration and Corporate Performance in the Czech Republic" } \\
\text { 27(3), Sept. 1999, pp. 498-513. }\end{array}$ & $\begin{array}{l}\text { Stijn Claessens and Simeon } \\
\text { Djankov }\end{array}$ & Apr. 1999 \\
\hline $\begin{array}{l}\text { No. 226: Published in Journal of Population Economics, "Poland on the } \\
\text { Dole: The Effect of Reducing the Unemployment Benefit Entitlement } \\
\text { Period during Transition" 13:35-44, } 2000 \text {. }\end{array}$ & Patrick A. Puhani & Mar. 1999 \\
\hline No. 225: Transition at Whirlpool-Tatramat: Case Studies & $\begin{array}{l}\text { Hans Brechbuhl and Sonia } \\
\text { Ferencikova }\end{array}$ & Mar. 1999 \\
\hline $\begin{array}{l}\text { No. 224: Measuring Progress in Transition and Towards EU Accession: } \\
\text { A Comparison of Manufacturing Firms in Poland, Romania, and Spain }\end{array}$ & $\begin{array}{l}\text { Wendy Carlin, Saul Estrin, and } \\
\text { Mark Schaffer }\end{array}$ & Mar. 1999 \\
\hline $\begin{array}{l}\text { No. 223: Product Market Competition in Transition Economies: } \\
\text { Increasing Varieties and Consumer Loyalty }\end{array}$ & Mitsutoshi M. Adachi & Mar. 1999 \\
\hline $\begin{array}{l}\text { No. 222: Opaque Markets and Rapid Growth: the Superiority of Bank- } \\
\text { Centered Financial Systems for Developing Nations }\end{array}$ & Rodney Wallace & July 1999 \\
\hline No. 221: Technology Spillovers through Foreign Direct Investment & Yuko Kinoshita & Jan. 1999 \\
\hline $\begin{array}{l}\text { No. 220: Managerial, Expertise and Team Centered Forms of } \\
\text { Organizing: A Cross-Cultural Exploration of Independence in } \\
\text { Engineering Work }\end{array}$ & Leslie Perlow & Jan. 1999 \\
\hline $\begin{array}{l}\text { No. 219: Household Structure and Labor Demand in Agriculture: } \\
\text { Testing for Separability in Rural China }\end{array}$ & $\begin{array}{l}\text { Audra J. Bowlus and Terry } \\
\text { Sicular }\end{array}$ & Jan. 1999 \\
\hline $\begin{array}{l}\text { No. 218: Competing Strategies of FDI and Technology Transfer to } \\
\text { China: American and Japanese Firms }\end{array}$ & $\begin{array}{l}\text { W. Mark Fruin and Penelope } \\
\text { Prime }\end{array}$ & Jan. 1999 \\
\hline $\begin{array}{l}\text { No. } 217 \text { Published in: Journal of Comparative Economics, "Returns to } \\
\text { Mobility in the Transition to a Market Economy" 27(1), Mar } 1999 .\end{array}$ & $\begin{array}{l}\text { Tito Boeri and Christopher J. } \\
\text { Flinn }\end{array}$ & Jan. 1999 \\
\hline $\begin{array}{l}\text { No. } 216 \text { Published in: Journal of Comparative Economics, "Labor } \\
\text { Market Policies and Unemployment in the Czech Republic." 27(1), Mar } \\
\text { 1999, pp. 33-60. }\end{array}$ & Katherine Terrell and Vit Sorm & Nov. 1998 \\
\hline $\begin{array}{l}\text { No. } 215 \text { Published in: Journal of Comparative Economics, "Active } \\
\text { Labor Market Policies in Poland: Human Capital Enhancement, } \\
\text { Stigmatization or Benefit Churning?" 27(1), Mar 1999, pp. 61-. }\end{array}$ & $\begin{array}{l}\text { Jochen Kluve, Hartmut Lehmann, } \\
\text { and Christoph M. Schmidt }\end{array}$ & Dec. 1998 \\
\hline
\end{tabular}




\begin{tabular}{|c|c|c|}
\hline $\begin{array}{l}\text { No. } 214 \text { Published in: Journal of Comparative Economics, "Does the } \\
\text { Slovenian Public Work Program Increase Participants' Chances to Find } \\
\text { a Job?" 27(1), Mar 1999, pp. 113- . }\end{array}$ & Milan Vodopivec & Dec. 1998 \\
\hline $\begin{array}{l}\text { No. } 213 \text { Published in: Journal of Comparative Economics, "Effects of } \\
\text { Active Labor Market Programs on the Transition Rate from } \\
\text { Unemployment into Regular Jobs in the Slovak Republic." 27(1), Mar } \\
\text { 1999, pp. 90- . }\end{array}$ & $\begin{array}{l}\text { Martina Lubyova and Jan C. van } \\
\text { Ours }\end{array}$ & Dec. 1998 \\
\hline $\begin{array}{l}\text { No. 212: The Marketing System in Bulgarian Livestock Production - } \\
\text { The Present State and Evolutionary Processes During the Period of } \\
\text { Economic Transition }\end{array}$ & Yordan Staykov, Team Leader & Oct. 1998 \\
\hline No. 211: Bankruptcy Experience in Hungary and the Czech Republic & Janet Mitchell & Oct. 1998 \\
\hline $\begin{array}{l}\text { No 210: Values, Optimum Stimulation Levels and Brand Loyalty: New } \\
\text { Scales in New Populations }\end{array}$ & $\begin{array}{l}\text { Steven M. Burgess and Mari } \\
\text { Harris }\end{array}$ & Sept. 1998 \\
\hline No. 209: Inherited Wealth, Corporate Control and Economic Growth & $\begin{array}{l}\text { Randall K. Morck, David A. } \\
\text { Stangeland, and Bernard Yeung }\end{array}$ & Sept. 1998 \\
\hline $\begin{array}{l}\text { No. 208: A Cultural Analysis of Homosocial Reproduction and } \\
\text { Contesting Claims to Competence in Transitional Firms }\end{array}$ & Michael D. Kennedy & July 1998 \\
\hline $\begin{array}{l}\text { No. 207: From Survival to Success: The Journey of Corporate } \\
\text { Transformation at Haier. Forthcoming in Managing Organizational } \\
\text { Change in Transition Economies ed. Daniel Denison. }\end{array}$ & $\begin{array}{l}\text { Arthur Yeung and Kenneth } \\
\text { DeWoskin }\end{array}$ & July 1998 \\
\hline $\begin{array}{l}\text { No. 206: Why Do People Work If They Are Not Paid? An Example } \\
\text { from Eastern Europe. Forthcoming in Managing Organizational Change } \\
\text { in Transition Economies. }\end{array}$ & Irina L. Zinovieva & May 1998 \\
\hline $\begin{array}{l}\text { No. 205: Firm Ownership and Work Motivation in Bulgaria and } \\
\text { Hungary: An Empirical Study of the Transition in the Mid-1990s. } \\
\text { Forthcoming in Managing Organizational Change in Transition } \\
\text { Economies ed. Daniel Denison. }\end{array}$ & $\begin{array}{l}\text { Robert A. Roe, Irina L. } \\
\text { Zinovieva, Elizabeth Dienes, and } \\
\text { Laurens A. ten Horn }\end{array}$ & May 1998 \\
\hline $\begin{array}{l}\text { No. 204: Human Resource Management in the Restructuring of Chinese } \\
\text { Joint Ventures. Forthcoming in Managing Organizational Change in } \\
\text { Transition Economies ed. Daniel Denison. }\end{array}$ & Nandani Lynton & Apr. 1998 \\
\hline $\begin{array}{l}\text { No. 203: Emergent Compensation Strategies in Post-Socialist Poland: } \\
\text { Understanding the Cognitive Underpinnings of Management Practices } \\
\text { in a Transition Economy. Forthcoming in Managing Organizational } \\
\text { Change in Transition Economies ed. Daniel Denison. }\end{array}$ & Marc Weinstein & Mar. 1998 \\
\hline $\begin{array}{l}\text { No. 202: Corporate Transformation and Organizational Learning: The } \\
\text { People's Republic of China. Forthcoming in Managing Organizational } \\
\text { Change in Transition Economies ed. Daniel Denison. }\end{array}$ & $\begin{array}{l}\text { Meinolf Dierkes and Zhang } \\
\text { Xinhua }\end{array}$ & Mar. 1998 \\
\hline $\begin{array}{l}\text { No. 201: Foreign Direct Investment as a Factor of Change: The Case of } \\
\text { Slovakia. Forthcoming in Managing Organizational Change in } \\
\text { Transition Economies ed. Daniel Denison. }\end{array}$ & Sonia Ferencikova & Feb. 1998 \\
\hline $\begin{array}{l}\text { No. 200: Radical versus Incremental Change: The Role of Capabilities, } \\
\text { Competition, and Leaders. Forthcoming in Managing Organizational } \\
\text { Change in Transition Economies ed. Daniel Denison. }\end{array}$ & Karen L. Newman & Feb. 1998 \\
\hline $\begin{array}{l}\text { No. 199: The Emergence of Market Practices in China's Economic } \\
\text { Transition: Price Setting Practices in Shanghai’s Industrial Firms. } \\
\text { Forthcoming in Managing Organizational Change in Transition } \\
\text { Economies ed. Daniel Denison. }\end{array}$ & Douglas Guthrie & Feb. 1998 \\
\hline $\begin{array}{l}\text { No. 198: The Application of Change Management Methods at Business } \\
\text { Organizations Operating in Hungary: Challenges in the Business and } \\
\text { Cultural Environment and First Practical Experiences. Forthcoming in } \\
\text { Managing Organizational Change in Transition Economies ed. Daniel } \\
\text { Denison. }\end{array}$ & Dr. János Fehér & Jan. 1998 \\
\hline $\begin{array}{l}\text { No. 197: Organizational Changes in Russian Industrial Enterprises: } \\
\text { Mutation of Decision-Making Structures and Transformations of } \\
\text { Ownership. Forthcoming in Managing Organizational Change in } \\
\text { Transition Economies ed. Daniel Denison. }\end{array}$ & Igor B. Gurkov & Jan. 1998 \\
\hline
\end{tabular}




\begin{tabular}{|c|c|c|}
\hline $\begin{array}{l}\text { No. 196: Understanding and Managing Challenges to the Romanian } \\
\text { Companies during Transition. Forthcoming in Managing Organizational } \\
\text { Change in Transition Economies ed. Daniel Denison. }\end{array}$ & $\begin{array}{l}\text { Dan Candea and Rodica M. } \\
\text { Candea }\end{array}$ & Jan. 1998 \\
\hline $\begin{array}{l}\text { No. 195: Insider Lending and Economic Transition: The Structure, } \\
\text { Function, and Performance Impact of Finance Companies in Chinese } \\
\text { Business Groups. Forthcoming in Managing Organizational Change in } \\
\text { Transition Economies ed. Daniel Denison. }\end{array}$ & Lisa A. Keister & Dec. 1997 \\
\hline $\begin{array}{l}\text { No. 194: Japanese Investment in Transitional Economies: } \\
\text { Characteristics and Performance. Forthcoming in Managing } \\
\text { Organizational Change in Transition Economies ed. Daniel Denison. }\end{array}$ & $\begin{array}{l}\text { Paul W. Beamish and Andrew } \\
\text { Delios }\end{array}$ & Nov. 1997 \\
\hline $\begin{array}{l}\text { No. 193: Building Successful Companies in Transition Economies. } \\
\text { Forthcoming in Managing Organizational Change in Transition } \\
\text { Economies ed. Daniel Denison. }\end{array}$ & Dr. Ivan Perlaki & Jan. 1998 \\
\hline $\begin{array}{l}\text { No. 192: Russian Communitariansim: An Invisible Fist in the } \\
\text { Transformation Process of Russia. Forthcoming in Managing } \\
\text { Organizational Change in Transition Economies ed. Daniel Denison. }\end{array}$ & Charalambos Vlachoutsicos & July 1998 \\
\hline No. 191: Teaching the Dinosaurs to Dance & Michal Cakrt & Sept. 1997 \\
\hline $\begin{array}{l}\text { No. 190: Strategic Restructuring: Making Capitalism in Post- } \\
\text { Communist Eastern Europe. Forthcoming in Managing Organizational } \\
\text { Change in Transition Economies ed. Daniel Denison. }\end{array}$ & Lawrence P. King & Sept. 1997 \\
\hline $\begin{array}{l}\text { No. 189: Published in: Regional Science and Urban Economics, } \\
\text { "Russia's Internal Border." 29(5), Sept. } 1999 .\end{array}$ & $\begin{array}{l}\text { Daniel Berkowitz and David N. } \\
\text { DeJong }\end{array}$ & July 1998 \\
\hline No. 187: Corporate Structure and Performance in Hungary & László Halpern and Gábor Kórsöi & July 1998 \\
\hline No. 186: Performance of Czech Companies by Ownership Structure & $\begin{array}{l}\text { Andrew Weiss and Georgiy } \\
\text { Nikitin }\end{array}$ & June 1998 \\
\hline $\begin{array}{l}\text { No. 185: Firm Performance in Bulgaria and Estonia: The effects of } \\
\text { competitive pressure, financial pressure and disorganisation }\end{array}$ & Jozef Konings & July 1998 \\
\hline $\begin{array}{l}\text { No. 184: Investment and Wages during the Transition: Evidence from } \\
\text { Slovene Firms }\end{array}$ & Janez Prasnikar and Jan Svejnar & July 1998 \\
\hline $\begin{array}{l}\text { No. 183: Investment Portfolio under Soft Budget: Implications for } \\
\text { Growth, Volatility and Savings }\end{array}$ & Chongen Bai and Yijiang Wang & July 1998 \\
\hline No. 181: Delegation and Delay in Bank Privatization & $\begin{array}{l}\text { Loránd Ambrus-Lakatos and } \\
\text { Ulrich Hege }\end{array}$ & July 1998 \\
\hline No. 180: Financing Mechanisms and R\&D Investment & $\begin{array}{l}\text { Haizhou Huang and Chenggang } \\
\mathrm{Xu}\end{array}$ & July 1998 \\
\hline $\begin{array}{l}\text { No. 179: Organizational Culture and Effectiveness: The Case of Foreign } \\
\text { Firms in Russia }\end{array}$ & $\begin{array}{l}\text { Carl F. Fey and Daniel R. } \\
\text { Denison }\end{array}$ & Jan. 1999 \\
\hline No. 178: Output and Unemployment Dynamics in Transition & $\begin{array}{l}\text { Vivek H. Dehejia and Douglas W. } \\
\text { Dwyer }\end{array}$ & Jan. 1998 \\
\hline $\begin{array}{l}\text { No. 177: Published in: Economics of Transition, "Bureaucracies in the } \\
\text { Russian Voucher Privatization." 8(1), 2000, pp. 37-57. }\end{array}$ & Guido Friebel & June 1998 \\
\hline No. 176: Chronic Moderate Inflation in Transition: The Tale of Hungary & János Vincze & June 1998 \\
\hline No. 175: Privatisation and Market Structure in a Transition Economy & John Bennett and James Maw & June 1998 \\
\hline $\begin{array}{l}\text { No. 174: Ownership and Managerial Competition: Employee, Customer, } \\
\text { or Outside Ownership }\end{array}$ & $\begin{array}{l}\text { Patrick Bolton and Chenggang } \\
\mathrm{Xu}\end{array}$ & June 1998 \\
\hline $\begin{array}{l}\text { No. 173: Intragovernment Procurement of Local Public Good: A } \\
\text { Theory of Decentralization in Nondemocratic Government }\end{array}$ & $\begin{array}{l}\text { Chong-en Bai, Yu Pan and } \\
\text { Yijiang Wang }\end{array}$ & June 1998 \\
\hline No. 172: Political Instability and Growth in Proprietary Economies & $\begin{array}{l}\text { Jody Overland and Michael } \\
\text { Spagat }\end{array}$ & Aug. 1998 \\
\hline $\begin{array}{l}\text { No. 171: Published in Post-Communist Economies, "Framework Issues } \\
\text { in the Privatization Strategies of the Czech Republic, Hungary, and } \\
\text { Poland" 11(1) Mar. } 1999 .\end{array}$ & Morris Bornstein & June 1998 \\
\hline $\begin{array}{l}\text { No. 170: Published in: European Journal of Political Economy } \\
\text { "Privatization, Ownership Structure and Transparency: How to Measure } \\
\text { a Real Involvement of the State" 15(4), Nov. 1999, pp. 605-18. }\end{array}$ & Frantisek Turnovec & May 1998 \\
\hline
\end{tabular}




\begin{tabular}{|c|c|c|}
\hline $\begin{array}{l}\text { No. } 169 \text { Published in: American Economic Review, "Unemployment and } \\
\text { the Social Safety Net during Transitions to a Market Economy: } \\
\text { Evidence from Czech and Slovak Men" 88(5), Dec 1998, pp. 1117-1142 }\end{array}$ & $\begin{array}{l}\text { John C. Ham, Jan Svejnar, and } \\
\text { Katherine Terrell }\end{array}$ & Dec. 1998 \\
\hline $\begin{array}{l}\text { No. 167: Voucher Privatization with Investment Funds: An Institutional } \\
\text { Analysis }\end{array}$ & David Ellerman & Mar. 1998 \\
\hline $\begin{array}{l}\text { No. 166: Published in: Marketing Issues in Transitional Economies, } \\
\text { "Value Priorities and Consumer Behavior in a Transitional Economy: } \\
\text { The Case of South Africa" ed. Rajeev Batra. }\end{array}$ & $\begin{array}{l}\text { Steven M. Burgess and Jan- } \\
\text { Benedict E.M. Steenkamp }\end{array}$ & Aug. 1998 \\
\hline $\begin{array}{l}\text { No. 164: Finance and Investment in Transition: Czech Enterprises, } \\
\text { 1993-1994 }\end{array}$ & $\begin{array}{l}\text { Ronald Anderson and Chantal } \\
\text { Kegels }\end{array}$ & Sept. 1997 \\
\hline $\begin{array}{l}\text { No. 163: European Union Trade and Investment Flows U-Shaping } \\
\text { Industrial Output in Central and Eastern Europe: Theory and Evidence }\end{array}$ & $\begin{array}{l}\text { Alexander Repkine and Patrick P. } \\
\text { Walsh }\end{array}$ & Apr. 1998 \\
\hline $\begin{array}{l}\text { No. 162: Skill Acquisition and Private Firm Creation in Transition } \\
\text { Economies }\end{array}$ & Zuzana Brixiova and Wenli Li & Oct. 1999 \\
\hline No. 161: Corruption in Transition & Susanto Basu and David D. Li & May 1998 \\
\hline $\begin{array}{l}\text { No. 160a: Tenures that Shook the World: Worker Turnover in Russia, } \\
\text { Poland and Britain }\end{array}$ & $\begin{array}{l}\text { Hartmut Lehmann and Jonathan } \\
\text { Wadsworth }\end{array}$ & Nov. 1999 \\
\hline $\begin{array}{l}\text { No. 160: Tenures that Shook the World: Worker Turnover in the } \\
\text { Russian Federation and Poland }\end{array}$ & $\begin{array}{l}\text { Hartmut Lehmann and Jonathan } \\
\text { Wadsworth }\end{array}$ & June 1998 \\
\hline No. 159: Does Market Structure Matter? New Evidence from Russia & $\begin{array}{l}\text { Annette N. Brown and J. David } \\
\text { Brown }\end{array}$ & June 1998 \\
\hline $\begin{array}{l}\text { No. 158: Structural Adjustment and Regional Long Term } \\
\text { Unemployment in Poland }\end{array}$ & $\begin{array}{l}\text { Hartmut Lehmann and Patrick P. } \\
\text { Walsh }\end{array}$ & June 1997 \\
\hline $\begin{array}{l}\text { No. 157: Baby Boom or Bust? Changing Fertility in Post-Communist } \\
\text { Czech Republic and Slovakia }\end{array}$ & Robert S. Chase & Apr. 1998 \\
\hline $\begin{array}{l}\text { No. } 156 \text { Published in: Leadership and Organization Development } \\
\text { Journal, "Leading Radical Change in Transition Economies." Vol. 19, } \\
\text { No. 6, 1998, pp. 309-324. }\end{array}$ & Karen L. Newman & June 1998 \\
\hline $\begin{array}{l}\text { No. } 155 \text { Published in: Oxford Review of Economic Policy, "From } \\
\text { Theory into Practice? Restructuring and Dynamism in Transition } \\
\text { Economies." Vol. 13, No. 2, Summer 1997, pp. 77-105. }\end{array}$ & $\begin{array}{l}\text { Wendy Carlin and Michael } \\
\text { Landesmann }\end{array}$ & June 1997 \\
\hline $\begin{array}{l}\text { No. 154: The Model and the Reality: Assessment of Vietnamese SOE } \\
\text { Reform-Implementation at the Firm Level }\end{array}$ & $\begin{array}{l}\text { Edmund Malesky, Vu Thanh } \\
\text { Hung, Vu Thi Dieu Anh, and } \\
\text { Nancy K. Napier }\end{array}$ & July 1998 \\
\hline $\begin{array}{l}\text { No. } 153 \text { Published in: Journal of Comparative Economics, "Causes of } \\
\text { the Soft Budget Constraint: Evidence on Three Explanations." Vol. 26, } \\
\text { No. 1, Mar. 1998, pp. 104-116. }\end{array}$ & David D. Li and Minsong Liang & Mar. 1998 \\
\hline $\begin{array}{l}\text { No. } 152 \text { Published in: Comparative Economic Studies, "Enterprise } \\
\text { Restructuring in Russia’s Transition Economy: Formal and Informal } \\
\text { Mechanisms." Vol. 40, No. 2, Summer 1998, pp. 5-52. }\end{array}$ & Susan J. Linz and Gary Krueger & Apr. 1998 \\
\hline $\begin{array}{l}\text { No. 151: Labor Productivity in Transition: A Regional Analysis of } \\
\text { Russian Industry }\end{array}$ & Susan J. Linz & May 1998 \\
\hline $\begin{array}{l}\text { No. 150: Tax Avoidance and the Allocation of Credit. Forthcoming in } \\
\text { Financial Systems in Transition: The Design of Financial Systems in } \\
\text { Central Europe eds. Anna Meyendorff and Anjan Thakor. }\end{array}$ & Anna Meyendorff & June 1998 \\
\hline $\begin{array}{l}\text { No. 149: Commitment, Versatility and Balance: Determinants of Work } \\
\text { Time Standards and Norms in a Multi-Country Study of Software } \\
\text { Engineers }\end{array}$ & Leslie Perlow and Ron Fortgang & Apr. 1998 \\
\hline $\begin{array}{l}\text { No. 148: Changes in Poland's Transfer Payments in the 1990s: the Fate } \\
\text { of Pensioners }\end{array}$ & Bozena Leven & June 1998 \\
\hline $\begin{array}{l}\text { No. 147: Environmental Protection and Economic Development: The } \\
\text { Case of the Huaihe River Basin Cleanup Plan }\end{array}$ & $\begin{array}{l}\text { Robert Letovsky, Reze Ramazani, } \\
\text { and Debra Murphy }\end{array}$ & June 1998 \\
\hline $\begin{array}{l}\text { No. 146: Chief Executive Compensation During Early Transition: } \\
\text { Further Evidence from Bulgaria }\end{array}$ & $\begin{array}{l}\text { Derek C. Jones, Takao Kato, and } \\
\text { Jeffrey Miller }\end{array}$ & June 1998 \\
\hline
\end{tabular}


No. 145 Published in: Economics of Transition, "Women's Unemployment During the Transition: Evidence from Czech and Slovak Micro Data," Vol. 7, No. 1, May 1999, pp. 47-78.

No. 144: Investment and Wages in Slovenia

No. 143 Published in: Review of Financial Studies, "Optimal

Bankruptcy Laws Across Different Economic Systems," 12(2), 47-77,

Summer 19993.

No. 142: Industrial Policy and Poverty in Transition Economies: Two

Steps Forward or One Step Back?

No. 141 Collective Ownership and Privatization of China's Village Enterprises

No. 140 A Comparative Look at Labor Mobility in the Czech Republic: Where have all the Workers Gone?

No. 139 The Failure of the Government-Led Program of Corporate

Reorganization in Romania

No. 138 Ownership and Employment in Russian Industry: 1992-1995

No. 137 Published in: Journal of Political Economy, "Reform Without

Losers: An Interpretation of China's Dual-Track Approach to

Transition," Feb. 2000; Vol. 108, Iss.1; pg. 120

No. 136 Published in: European Economic Review, "The Political

Economy of Mass Privatization and the Risk of Expropriation," 44(2),

Feb. 2000, pgs. 393-421

No. 135: Radical Organizational Change: The Role of Starting

Conditions, Competition, and Leaders

No. 134: To Restructure or Not to Restructure: Informal Activities and

Enterprise Behavior in Transition

No. 133: Management 101: Behavior of Firms in Transition Economies

No. 132 Published in: Quarterly Journal of Economics, "Interfirm

Relationships and Informal Credit in Vietnam," 114(4), Nov. 1999, pgs.

$1285-1320$

No. 131 Published in: Comparative Economic Studies, "Will

Restructuring Hungarian Companies Innovate? An Investigation Based

on Joseph Berliner's Analysis of Innovation in Soviet Industry." Vol.

40, No. 2, Summer 1998, pp. 53-74.

No. 130: Published in The American Economic Review, "Changing

Incentives of the Chinese Bureaucracy." May, 1998.

No. 129: Restructuring Investment in Transition: A Model of the

Enterprise Decision

No. 128 Published in: Comparative Economic Studies, "Job Rights in

Russian Firms: Endangered or Extinct Institutions?” Vol. 40, No. 4,

Winter 1998, pp. 1-32.

No. 127: Accounting for Growth in Post-Soviet Russia

No. 126 Published in: Economics of Transition, "From Federalism,

Chinese Style, to Privatization Chinese Style," 7(1), 1999, pgs. 103-31

No. 125: Market Discipline in Conglomerate Banks: Is an Internal

Allocation of Cost of Capital Necessary as Incentive Device?

Forthcoming in Financial Systems in Transition: The Design of

Financial Systems in Central Europe eds. Anna Meyendorff and Anjan

Thakor.

No. 124: Financial Discipline in the Enterprise Sector in Transition

Countries: How Does China Compare?

No. 123: Considerations of an Emerging Marketplace: Managers'

Perceptions in the Southern African Economic Community

No. 122: A Model of the Informal Economy in Transition Economies

\begin{tabular}{|c|c|}
\hline $\begin{array}{l}\text { John Ham, Jan Svejnar, and } \\
\text { Katherine Terrell }\end{array}$ & May 1998 \\
\hline Janez Prasnikar & May 1998 \\
\hline $\begin{array}{l}\text { Elazar Berkovitch and Ronen } \\
\text { Israel }\end{array}$ & Mar. 1998 \\
\hline Susan J. Linz & Mar. 1998 \\
\hline Suwen Pan and Albert Park & Apr. 1998 \\
\hline Vit Sorm and Katherine Terrell & Apr. 1999 \\
\hline $\begin{array}{l}\text { Simeon Djankov and Kosali } \\
\text { Ilayperuma }\end{array}$ & Sept. 1997 \\
\hline Susan J. Linz & Mar. 1998 \\
\hline $\begin{array}{l}\text { Lawrence J. Lau, Yingyi Qian, } \\
\text { and Gerard Roland }\end{array}$ & Nov. 1997 \\
\hline Klaus M. Schmidt & Mar. 1998 \\
\hline Karen L. Newman & Jan. 1998 \\
\hline $\begin{array}{l}\text { Clifford Gaddy and Barry W. } \\
\text { Ickes }\end{array}$ & May 1998 \\
\hline Josef C. Brada & Mar. 1998 \\
\hline $\begin{array}{l}\text { John McMillan and Christopher } \\
\text { Woodruff }\end{array}$ & Feb. 1998 \\
\hline John B. Bonin and Istvan Abel & Mar. 1998 \\
\hline David D. Li & Jan. 1998 \\
\hline Richard E. Ericson & Jan. 1998 \\
\hline Susan J. Linz & Jan. 1998 \\
\hline $\begin{array}{l}\text { Daniel Berkowitz and David N. } \\
\text { DeJong }\end{array}$ & Jan. 1998 \\
\hline $\begin{array}{l}\text { Yuanzheng Cao, Yingyi Qian, } \\
\text { and Barry R. Weingast }\end{array}$ & Dec. 1997 \\
\hline $\begin{array}{l}\text { Arnoud W. A. Boot and Anjolein } \\
\text { Schmeits }\end{array}$ & Nov. 1997 \\
\hline $\begin{array}{l}\text { Shumei Gao and Mark E. } \\
\text { Schaffer }\end{array}$ & Feb. 1998 \\
\hline Brent Chrite and David Hudson & Feb. 1998 \\
\hline $\begin{array}{l}\text { Simon Commander and Andrei } \\
\text { Tolstopiatenko }\end{array}$ & Nov. 1997 \\
\hline
\end{tabular}




\begin{tabular}{|c|c|c|}
\hline $\begin{array}{l}\text { No. 121: Local Labour Market Dynamics in the Czech and Slovak } \\
\text { Republics }\end{array}$ & $\begin{array}{l}\text { Peter Huber and Andreas } \\
\text { Worgotter }\end{array}$ & Nov. 1997 \\
\hline $\begin{array}{l}\text { No. 119: Published in Academy of Management Review, “Organizational } \\
\text { Transformation during Institutional Upheaval," } 25 \text { (3), 2000, p. 602-619 }\end{array}$ & Karen L. Newman & Mar. 1998 \\
\hline No. 118: Industrial Decline and Labor Reallocation in Romania & John S. Earle & Oct. 1997 \\
\hline No. 117: Notes for an Essay on the Soft Budget Constraint & Lorand Ambrus-Lakatos & Jan. 1997 \\
\hline No. 116: Labor Demand During Transition in Hungary & Gabor Korosi & Oct. 1997 \\
\hline No. 115: Enterprise Performance and Managers' Profiles & $\begin{array}{l}\text { Simeon Djankov and Stijn } \\
\text { Claessens }\end{array}$ & Dec. 1997 \\
\hline $\begin{array}{l}\text { No. 114b Employment and Wages in Enterprises under Communism } \\
\text { and in Transition: Evidence From Central Europe and Russia }\end{array}$ & $\begin{array}{l}\text { Swati Basu, Saul Estrin, and Jan } \\
\text { Svejnar }\end{array}$ & Apr. 2000 \\
\hline $\begin{array}{l}\text { No. 114: Employment and Wage Behavior of Enterprises in Transitional } \\
\text { Economies }\end{array}$ & $\begin{array}{l}\text { Swati Basu, Saul Estrin, and Jan } \\
\text { Svejnar }\end{array}$ & Oct. 1997 \\
\hline $\begin{array}{l}\text { No. 113: Preliminary Evidence on Active Labor Programs' Impact in } \\
\text { Hungary and Poland }\end{array}$ & Christopher J. O'Leary & Oct. 1997 \\
\hline $\begin{array}{l}\text { No. 111: Unemployment Benefits and Incentives in Hungary: New } \\
\text { Evidence }\end{array}$ & Joachim Wolff & Oct. 1997 \\
\hline $\begin{array}{l}\text { No. 110: Published in: Empirical Economics, "Long-Term } \\
\text { Unemployment, Unemployment Benefits and Social Assistance: The } \\
\text { Polish Experience" Empirical-Economics; 23(1-2), 1998, pp. 55-85. }\end{array}$ & $\begin{array}{l}\text { Marek Gora and Christoph M. } \\
\text { Schmidt }\end{array}$ & Apr. 1997 \\
\hline $\begin{array}{l}\text { No. } 109 \text { Published in: Industrial and Labor Relations Review, "Markets } \\
\text { for Communist Human Capital: Returns to Education and Experience in } \\
\text { Post-Communist Czech Republic and Slovakia." 51(3), Apr. 1998, pp. } \\
\text { 401-423. }\end{array}$ & Robert S. Chase & Oct. 1997 \\
\hline $\begin{array}{l}\text { No. 107: The Worker-Firm Matching in the Transition: (Why) Are the } \\
\text { Czechs More Successful Than Others? }\end{array}$ & $\begin{array}{l}\text { Daniel Münich, Jan Svejnar, and } \\
\text { Katherine Terrell }\end{array}$ & Oct. 1997 \\
\hline $\begin{array}{l}\text { No. } 106 \text { Published in: Journal of Comparative Economics, "Job } \\
\text { Creation, Job Destruction and Growth of Newly Established, Privatized } \\
\text { and State-Owned Enterprises in Transition Economies: Survey Evidence } \\
\text { from Bulgaria, Hungary, and Romania," 26(3), Sept. 1998, pp. 429-445. }\end{array}$ & $\begin{array}{l}\text { Valentijn Bilsen and Jozef } \\
\text { Konings }\end{array}$ & Sept. 1998 \\
\hline $\begin{array}{l}\text { No. 105: Getting Behind the East-West [German] Wage Differential: } \\
\text { Theory and Evidence }\end{array}$ & $\begin{array}{l}\text { Michael Burda and Christoph } \\
\text { Schmidt }\end{array}$ & May 1997 \\
\hline No. 104: The Birth of the "Wage Curve" in Hungary, 1989-95 & Gabor Kertesi and Janos Kollo & Oct. 1997 \\
\hline $\begin{array}{l}\text { No. 103: Published in: Journal of Comparative Economics, "Grime and } \\
\text { Punishment: Job Insecurity and Wage Arrears in the Russian } \\
\text { Federation" 27, 595-617 (1999). }\end{array}$ & $\begin{array}{l}\text { Hartmut Lehmann, Jonathan } \\
\text { Wadsworth, and Alessandro } \\
\text { Acquisti }\end{array}$ & Oct. 1997 \\
\hline No. 102: Social Networks in Transition & $\begin{array}{l}\text { Lorena Barberia, Simon Johnson, } \\
\text { and Daniel Kaufmann }\end{array}$ & Oct. 1997 \\
\hline $\begin{array}{l}\text { No. 101: Depreciation and Russian Corporate Finance: A Pragmatic } \\
\text { Approach to Surviving the Transition }\end{array}$ & Susan J. Linz & Nov. 1997 \\
\hline No. 100: Romanian Financial System Reform & $\begin{array}{l}\text { Anna Meyendorff and Anjan V. } \\
\text { Thakor }\end{array}$ & Nov. 1997 \\
\hline $\begin{array}{l}\text { No. 99: Proceedings of the Conference on Strategic Alliances in } \\
\text { Transitional Economies, held May 20, } 1997 \text { at the Davidson Institute }\end{array}$ & Edited by Cynthia Koch & May 1997 \\
\hline No. 98: Institutions, Strain and the Underground Economy & Daniel Daianu and Lucian Albu & Nov. 1997 \\
\hline No. 97: Structure and Strain in Explaining Inter-Enterprise Arrears & Daniel Daianu & Nov. 1997 \\
\hline $\begin{array}{l}\text { No. 96: Resource Misallocation and Strain: Explaining Shocks in Post- } \\
\text { Command Economies }\end{array}$ & Daniel Daianu & Nov. 1997 \\
\hline $\begin{array}{l}\text { No. 95: Published in: Finance-a-Uver, "Czech Money Market: Emerging } \\
\text { Links Among Interest Rates." 48(2) } 1998 \text { pp. 99-109. }\end{array}$ & $\begin{array}{l}\text { Jan Hanousek and Evzen } \\
\text { Kocenda }\end{array}$ & Nov. 1997 \\
\hline $\begin{array}{l}\text { No. 94: Pre-Reform Industry and the } \\
\text { State Monopsony in China }\end{array}$ & $\begin{array}{l}\text { Xiao-Yuan Dong and Louis } \\
\text { Putterman }\end{array}$ & Oct. 1997 \\
\hline $\begin{array}{l}\text { No. 93: China's State-Owned Enterprises } \\
\text { In the First Reform Decade: } \\
\text { An Analysis of a Declining Monopsony }\end{array}$ & $\begin{array}{l}\text { Xiao-Yuan Dong and Louis } \\
\text { Putterman }\end{array}$ & Oct. 1997 \\
\hline No. 92: Expatriate Management in the Czech Re & Richard B. Peterson & Sept. 1997 \\
\hline
\end{tabular}




\begin{tabular}{|c|c|c|}
\hline No. 91: China and the Idea of Economic Reform & Thomas G. Rawski & Apr. 1997 \\
\hline $\begin{array}{l}\text { No. } 90 \text { Published in: China Economic Review, “China's State Enterprise } \\
\text { Reform: An Overseas Perspective.” Vol. 8, Spring 1997, pp. 89-98. }\end{array}$ & Thomas G. Rawski & July 1997 \\
\hline $\begin{array}{l}\text { No. 89: The Economic Determinants of Internal Migration Flows in } \\
\text { Russia During Transition }\end{array}$ & Annette N. Brown & July 1997 \\
\hline $\begin{array}{l}\text { No. 88: Gender Wage Gaps in China's Labor Market: Size, Structure, } \\
\text { Trends }\end{array}$ & $\begin{array}{l}\text { Margaret Maurer-Fazio, Thomas } \\
\text { G. Rawski, and Wei Zhang }\end{array}$ & July 1997 \\
\hline No. 87: Privatisation in Central and Eastern Europe & Saul Estrin & June 1997 \\
\hline $\begin{array}{l}\text { No. 86: Published in : Economics of Transition, "The Effect of } \\
\text { Privatization on Wealth Distribution in Russia." v. 7, no. 2, 1999, pp. } \\
449-65\end{array}$ & Michael Alexeev & Feb. 1998 \\
\hline $\begin{array}{l}\text { No. 85: Was Privatization in Eastern Germany a Special Case? Some } \\
\text { Lessons from the Treuhand }\end{array}$ & Uwe Siegmund & Sept. 1997 \\
\hline No. 84: Start-ups and Transition & $\begin{array}{l}\text { Daniel M. Berkowitz and David J. } \\
\text { Cooper }\end{array}$ & Sept. 1997 \\
\hline $\begin{array}{l}\text { No. 83: Which Enterprises (Believe They) Have Soft Budgets after } \\
\text { Mass Privatization? Evidence from Mongolia }\end{array}$ & $\begin{array}{l}\text { James Anderson, Georges } \\
\text { Korsun, and Peter Murrell }\end{array}$ & Oct. 1997 \\
\hline $\begin{array}{l}\text { No. 82: Published in: European Economic Review, "Unemployment } \\
\text { Dynamics and the Restructuring of the Slovak Unemployment Benefit } \\
\text { System." Apr., } 1997 .\end{array}$ & $\begin{array}{l}\text { Martina Lubyova and Jan C. van } \\
\text { Ours }\end{array}$ & June 1997 \\
\hline No. 81: Determinants of Unemployment Duration in Russia & Mark C. Foley & Aug. 1997 \\
\hline No. 80: The Many Faces of Information Disclosure & $\begin{array}{l}\text { Arnoud W.A. Boot and Anjan V. } \\
\text { Thakor }\end{array}$ & Oct. 1997 \\
\hline $\begin{array}{l}\text { No. 79: Published in: Journal of Finance, "Foreign Speculators and } \\
\text { Emerging Equity Markets."v.22, iss. 2, 2000, pp. 565-613 }\end{array}$ & $\begin{array}{l}\text { Geert Bekaert and Campbell R. } \\
\text { Harvey }\end{array}$ & Aug. 1997 \\
\hline $\begin{array}{l}\text { No. 78: The Relationship Between Economic Factors and Equity } \\
\text { Markets in Central Europe }\end{array}$ & $\begin{array}{l}\text { Jan Hanousek and Randall K. } \\
\text { Filer }\end{array}$ & June 1997 \\
\hline $\begin{array}{l}\text { No. } 77 \text { Published in: Economics of Transition, "A Gini Decomposition } \\
\text { Analysis of Inequality in the Czech and Slovak Republics During the } \\
\text { Transition," Vol. 6, No.1, May 1998, pp. 23-46. }\end{array}$ & $\begin{array}{l}\text { Thesia I. Garner and Katherine } \\
\text { Terrell }\end{array}$ & May 1998 \\
\hline $\begin{array}{l}\text { No. 76: China's Emerging Market for Property Rights: Theoretical and } \\
\text { Empirical Perspectives }\end{array}$ & $\begin{array}{l}\text { Gary H. Jefferson and Thomas G. } \\
\text { Rawski }\end{array}$ & June 1997 \\
\hline $\begin{array}{l}\text { No. 75b: Test of Permanent Income Hypothesis on Czech Voucher } \\
\text { Privatization }\end{array}$ & Jan Hanousek and Zdenek Tima & Oct. 1997 \\
\hline $\begin{array}{l}\text { No. 74: Determinants of Performance of Manufacturing Firms in Seven } \\
\text { European Transition Economies }\end{array}$ & $\begin{array}{l}\text { Stijn Claessens, Simeon Djankov, } \\
\text { and Gerhard Pohl }\end{array}$ & Feb. 1997 \\
\hline $\begin{array}{l}\text { No. } 73 \text { Published in: Economics of Transition, "The Restructuring of } \\
\text { Large Firms in Slovak Republic." Vol. 6, No. 1, May 1998, pp. 67-85 }\end{array}$ & $\begin{array}{l}\text { Simeon Djankov and Gerhard } \\
\text { Pohl }\end{array}$ & May 1998 \\
\hline $\begin{array}{l}\text { No. 72: Law, Relationships, and Private Enforcement: Transactional } \\
\text { Strategies of Russian Enterprises }\end{array}$ & $\begin{array}{l}\text { Kathryn Hendley, Peter Murrell, } \\
\text { and Randi Ryterman }\end{array}$ & Nov. 1998 \\
\hline $\begin{array}{l}\text { No. 71: Giving Credit Where Credit Is Due: The Changing Role of } \\
\text { Rural Financial Institutions in China }\end{array}$ & $\begin{array}{l}\text { Albert Park, Loren Brandt, and } \\
\text { John Giles }\end{array}$ & Mar. 1997 \\
\hline $\begin{array}{l}\text { No. 70: Privatization Versus Competition: Changing Enterprise } \\
\text { Behavior in Russia }\end{array}$ & John S. Earle and Saul Estrin & $\begin{array}{l}\text { Spring } \\
1997\end{array}$ \\
\hline $\begin{array}{l}\text { No. 69: Russian Managers under Storm: Explicit Reality and Implicit } \\
\text { Leadership Theories (A Pilot Exploration) }\end{array}$ & Igor Gurkov & Oct. 1998 \\
\hline $\begin{array}{l}\text { No. 68: The Political Economy of Central-Local Relations in China: } \\
\text { Inflation and Investment Controls During the Reform Era }\end{array}$ & Yasheng Huang & $\begin{array}{l}\text { Spring } \\
1997\end{array}$ \\
\hline $\begin{array}{l}\text { No. 67: Between Two Coordination Failures: Automotive Industrial } \\
\text { Policy in China with a Comparison to Korea }\end{array}$ & Yasheng Huang & $\begin{array}{l}\text { Spring } \\
1997\end{array}$ \\
\hline $\begin{array}{l}\text { No. } 66 \text { Published in: Post-Soviet Geography and Economics, "Red } \\
\text { Executives in Russia's Transition Economy." Vol. 27, No. 10, Nov. } \\
\text { 1996, pp. 633-651. }\end{array}$ & Susan J. Linz & Jan. 1997 \\
\hline $\begin{array}{l}\text { No. } 65 \text { Published in: Industrial and Corporate Change, "On the } \\
\text { Sequencing of Privatization in Transition Economies." Vol. 7, No. 1, } \\
1998 .\end{array}$ & $\begin{array}{l}\text { Gautam Ahuja and Sumit K. } \\
\text { Majumdar }\end{array}$ & Apr. 1997 \\
\hline
\end{tabular}




\begin{tabular}{|c|c|c|}
\hline $\begin{array}{l}\text { No. 64: Published in: Journal of Law and Economics, "Foreign } \\
\text { Ownership and Profitability: Property Rights, Control and the } \\
\text { Performance of Firms in Indian Industry" 42(1), Apr. 1999, pp. 209-38. }\end{array}$ & $\begin{array}{l}\text { Pradeep K. Chhibber and Sumit } \\
\text { K. Majumdar }\end{array}$ & Apr. 1997 \\
\hline No. 63: How Taxing Is Corruption on International Investors? & Shang-Jin Wei & Feb. 1997 \\
\hline $\begin{array}{l}\text { No. 62: What Can We Learn from the Experience of Transitional } \\
\text { Economies with Labour Market Policies? }\end{array}$ & Tito Boeri & 1997 \\
\hline $\begin{array}{l}\text { No. 61: Published in: Accounting Organizations and Society, } \\
\text { "Economic Transition, Strategy and the Evolution of Management } \\
\text { Accounting Practices: The Case of India" 24(5,6), Jul/Aug 1999, pp. } \\
\text { 379-412. }\end{array}$ & $\begin{array}{l}\text { Shannon W. Anderson and } \\
\text { William N. Lanen }\end{array}$ & Apr. 1997 \\
\hline $\begin{array}{l}\text { No. 60a: Enterprise Investment During the Transition: Evidence from } \\
\text { Czech Panel Data }\end{array}$ & Lubomír Lizal and Jan Svejnar & Dec. 1997 \\
\hline $\begin{array}{l}\text { No. 59: Published in: Journal of Law, Economics, and Organization, } \\
\text { "Institutional Environment, Community Government, and Corporate } \\
\text { Governance: Understanding China's Township-Village Enterprises." } \\
\text { 14(1), Apr. 1998, pages 1-23 }\end{array}$ & Jiahua Che and Yingyi Qian & Apr. 1997 \\
\hline No. 58: From the Grabbing Hand to the Helping Hand & Jiahua Che & June 2000 \\
\hline $\begin{array}{l}\text { No. 57: Published in: Brookings Papers on Economic Activity, "The } \\
\text { Unofficial Economy in Transition." 1: } 1998 .\end{array}$ & $\begin{array}{l}\text { Simon Johnson, Daniel } \\
\text { Kaufmann, and Andrei Schleifer }\end{array}$ & June 1997 \\
\hline No. 56: Taxes and Government Incentives: Eastern Europe vs. China & Roger H. Gordon and David D. Li & Apr. 1997 \\
\hline No. 55: Corruption and Reform & Susanto Basu and David Li & June 1996 \\
\hline $\begin{array}{l}\text { No. 54: Decentralization and the Macroeconomic Consequences of } \\
\text { Commitment to State-Owned Firms }\end{array}$ & Loren Brandt and Xiaodong Zhu & June 1997 \\
\hline $\begin{array}{l}\text { No. 53: Published in: The International Journal of Industrial } \\
\text { Organization, "Competitive Shocks and Industrial Structure: The Case } \\
\text { of Polish Manufacturing." Aug., 1999. . }\end{array}$ & $\begin{array}{l}\text { Pankaj Ghemawat and Robert E. } \\
\text { Kennedy }\end{array}$ & May 1997 \\
\hline $\begin{array}{l}\text { No. 52: Published in: The Quarterly Journal of Economics, "Insecure } \\
\text { Property Rights and Government Ownership of Firms." May, } 1998 .\end{array}$ & Jiahua Che and Yingyi Qian & May 1997 \\
\hline No. 51: Incentives, Scale Economies, and Organizational Form & $\begin{array}{l}\text { Eric Maskin, Yingyi Qian, and } \\
\text { Chenggang Xu }\end{array}$ & May 1997 \\
\hline $\begin{array}{l}\text { No. 50: Published in: Post-Soviet-Affairs, "End of the Tunnel? The } \\
\text { Effects of Financial Stabilization in Russia" Apr.-June 1997, pages 105- } \\
33\end{array}$ & $\begin{array}{l}\text { Barry W. Ickes, Peter Murrell, } \\
\text { and Randi Ryterman }\end{array}$ & Mar. 1997 \\
\hline $\begin{array}{l}\text { No. 49: The Evolution of Bank Credit Quality in Transition: Theory and } \\
\text { Evidence from Romania }\end{array}$ & $\begin{array}{l}\text { Enrico C. Perotti and Octavian } \\
\text { Carare }\end{array}$ & Oct. 1996 \\
\hline $\begin{array}{l}\text { No. 48: Where Do the Leaders Trade? Information Revelation and } \\
\text { Interactions Between the Segments of Czech Capital Markets }\end{array}$ & $\begin{array}{l}\text { Jan Hanousek and Libor } \\
\text { Nemecek }\end{array}$ & May 1997 \\
\hline $\begin{array}{l}\text { No. 47: Firms' Heterogeneity in Transition: Evidence from a Polish } \\
\text { Panel Data Set }\end{array}$ & $\begin{array}{l}\text { Irena Grosfeld and Jean-François } \\
\text { Nivet } \\
\text { Janet Mitchell }\end{array}$ & May 1997 \\
\hline No. 46: Strategic Creditor Passivity, Regulation, and Bank Bailouts & Janet Mitchell & May 1997 \\
\hline $\begin{array}{l}\text { No. 45a: Published in: Journal of Public Economics, "Tax Rights in } \\
\text { Transition Economies: A Tragedy of the Commons.” 76, 2000, pp. 369- } \\
397\end{array}$ & Daniel M. Berkowitz and Wei Li & Sept. 1997 \\
\hline $\begin{array}{l}\text { No. 44a: The Information Content of Stock Markets: Why do Emerging } \\
\text { Markets have Synchronous Stock Price Movements? (forthcoming in } \\
\text { the Journal of Financial Economics). }\end{array}$ & $\begin{array}{l}\text { Randall Morck, Bernard Yeung, } \\
\text { and Wayne Yu }\end{array}$ & Feb. 1999 \\
\hline $\begin{array}{l}\text { No. 43: Agency in Project Screening and Termination Decisions: Why } \\
\text { Is Good Money Thrown After Bad? }\end{array}$ & Chong-en Bai and Yijiang Wang & May 1997 \\
\hline $\begin{array}{l}\text { No. 42: Published in: Economics of Transition, "Channels of } \\
\text { Redistribution: Inequality and Poverty in the Russian Transition." Vol. } 7 \\
\text { (2) } 1999 .\end{array}$ & $\begin{array}{l}\text { Simon Commander, Andrei } \\
\text { Tolstopiatenko, and Ruslan } \\
\text { Yemtsov }\end{array}$ & May 1997 \\
\hline $\begin{array}{l}\text { No. 41: Published in: Economics of Transition, "Labour Market } \\
\text { Characteristics and Profitability: Econometric Analysis of Hungarian } \\
\text { Exporting Firms, 1986-1995" 6(1), May 1998, pages 145-62 }\end{array}$ & László Halpern and Gabor Korosi & May 1997 \\
\hline
\end{tabular}




\begin{tabular}{|c|c|c|}
\hline $\begin{array}{l}\text { No. 40: Published in: the Harvard Law Review, "The Tragedy of the } \\
\text { Anticommons: Property in the Transition from Marx to Markets." Jan. } \\
\text { 1998. }\end{array}$ & Michael Heller & Feb. 1997 \\
\hline No. 39: Privatization and Managerial Efficiency & $\begin{array}{l}\text { Olivier Debande and Guido } \\
\text { Friebel }\end{array}$ & May 1997 \\
\hline $\begin{array}{l}\text { No. } 38 \text { Published in: The Quarterly Journal of Economics, } \\
\text { "Disorganization." Vol. 112, No. 4, Nov. 1997, pp. 1091-1126. }\end{array}$ & $\begin{array}{l}\text { Olivier Blanchard and Michael } \\
\text { Kremer }\end{array}$ & Jan. 1997 \\
\hline $\begin{array}{l}\text { No. 37: Published in: Economics of Transition, "Transition and the } \\
\text { Output Fall." } 7(1), 1999 \text {, pages 1-28. }\end{array}$ & $\begin{array}{l}\text { Gérard Roland and Thierry } \\
\text { Verdier }\end{array}$ & Mar. 1997 \\
\hline $\begin{array}{l}\text { No. 36: Restructuring an Industry During Transition: A Two-Period } \\
\text { Model }\end{array}$ & Richard Ericson & Sept. 1996 \\
\hline No. 34: The East-West Joint Venture: BC Torsion Case Study & $\begin{array}{l}\text { Sonia Ferencikova and Vern } \\
\text { Terpstra }\end{array}$ & Dec. 1998 \\
\hline $\begin{array}{l}\text { No. } 33 \text { Published in: Journal of Comparative Economics, "Quantifying } \\
\text { Price Liberalization in Russia." Vol. 26, No. 4, Dec. 1998, pp. 735-737. }\end{array}$ & $\begin{array}{l}\text { Daniel Berkowitz, David DeJong, } \\
\text { and Steven Husted }\end{array}$ & Dec. 1998 \\
\hline No. 32: What Can North Korea Learn from China's Market Reforms? & John McMillan & Sept. 1996 \\
\hline $\begin{array}{l}\text { No. 31: Published in: China-Economic-Review, "Towards a Model of } \\
\text { China as a Partially Reformed Developing Economy Under a } \\
\text { Semifederalist Government." 9(1), Spring 1998, pages 1-23. }\end{array}$ & Yijiang Wang and Chun Chang & Mar. 1997 \\
\hline $\begin{array}{l}\text { No. 30: Convergence in Output in Transition Economies: Central and } \\
\text { Eastern Europe, 1970-1995 }\end{array}$ & Saul Estrin and Giovanni Urga & Feb. 1997 \\
\hline $\begin{array}{l}\text { No. 29: Published in: Economics of Transition, "Altered Band and } \\
\text { Exchange Volatility." Volume 6, no. 1, 1998, 173-181. }\end{array}$ & Evzen Kocenda & Mar. 1997 \\
\hline $\begin{array}{l}\text { No. 28: Published in: Quarterly Journal of Economics, "Public Versus } \\
\text { Private Ownership of Firms: Evidence from Rural China." Volume 113, } \\
\text { no. 3, Aug. 1998, 773-808. }\end{array}$ & Hehui Jin and Yingyi Qian & Jan. 1997 \\
\hline $\begin{array}{l}\text { No. 27: East-West Joint Ventures in a Transitional Economy: The Case } \\
\text { of Slovakia }\end{array}$ & Sonia Ferencikova & Mar. 1997 \\
\hline $\begin{array}{l}\text { No. 26: Published in Economic Analysis "Behavior of a Slovenian Firm } \\
\text { in Transition" Vol. 1, no. 1, 1998, 57-73. }\end{array}$ & Janez Prasnikar & Feb. 1997 \\
\hline $\begin{array}{l}\text { No. 25: Cultural Encounters and Claims to Expertise in Postcommunist } \\
\text { Capitalism }\end{array}$ & Michael D. Kennedy & Feb. 1997 \\
\hline $\begin{array}{l}\text { No. 24: ZVU a.s.: Investment Funds on the Board of Directors of an } \\
\text { Engineering Giant }\end{array}$ & Tory Wolff & Aug. 1995 \\
\hline $\begin{array}{l}\text { No. 23: The Role of Investment Funds in the Czech Republic (joint } \\
\text { publication with Czech Management Center) }\end{array}$ & Dusan Triska & June 1996 \\
\hline $\begin{array}{l}\text { No. 22: Czech Investment Fund Industry: Development and Behaviour } \\
\text { (joint publication with Czech Management Center) }\end{array}$ & Richard Podpiera & May 1996 \\
\hline $\begin{array}{l}\text { No. 21: Restructuring of Czech Firms: An Example of Gama, a.s. (joint } \\
\text { publication with Czech Management Center) }\end{array}$ & Antonin Bulin & June 1996 \\
\hline $\begin{array}{l}\text { No. 20: YSE Funds: A Story of Czech Investment Funds (joint } \\
\text { publication with Czech Management Center) }\end{array}$ & Michal Otradovec & Nov. 1995 \\
\hline $\begin{array}{l}\text { No. 19: První Investicni a.s., The First Investment Corporation (joint } \\
\text { publication with Czech Management Center) }\end{array}$ & Jaroslav Jirasek & Aug. 1995 \\
\hline $\begin{array}{l}\text { No. 18: PPF a.s., The First Private Investment Fund (joint publication } \\
\text { with Czech Management Center) }\end{array}$ & Michal Otradovec & Nov. 1995 \\
\hline $\begin{array}{l}\text { No. } 17 \text { Published in: Post-Soviet Geography and Economics, "Russia's } \\
\text { Managers in Transition: Pilferers or Paladins?" 37(7) (Sept. 1996), pp. } \\
\text { 397-426. }\end{array}$ & Susan J. Linz and Gary Krueger & Nov. 1996 \\
\hline $\begin{array}{l}\text { No. 16: Banks in Transition-Investment Opportunities in Central } \\
\text { Europe and Russia, Edited Transcript from } 31 \text { May } 1996 \text { Conference in } \\
\text { New York City }\end{array}$ & $\begin{array}{l}\text { With commentary and edited by } \\
\text { Anna Meyendorff }\end{array}$ & Jan. 1997 \\
\hline $\begin{array}{l}\text { No. 15: Marketing in Transitional Economies: Edited Transcript \& } \\
\text { Papers from } 1 \text { Apr. } 1996 \text { Conference in Ann Arbor, Michigan }\end{array}$ & $\begin{array}{l}\text { Compiled by The Davidson } \\
\text { Institute }\end{array}$ & Dec. 1996 \\
\hline
\end{tabular}


Davidson Institute Working Papers are available at: www.wdi.bus.umich.edu

\begin{tabular}{|c|c|c|c|}
\hline \multicolumn{2}{|c|}{$\begin{array}{l}\text { No. 14: Pensions in the Former Soviet Bloc: Problems and Solutions. } \\
\text { Published by Council on Foreign Relations. "The Coming Global } \\
\text { Pension Crisis" New York, } 1997\end{array}$} & Jan Svejnar & Nov. 1996 \\
\hline \multicolumn{2}{|c|}{$\begin{array}{l}\text { No. 13: Enterprise Restructuring and Performance in the Transition. } \\
\text { Forthcoming in Financial Systems in Transition: The Design of } \\
\text { Financial Systems in Central Europe eds. Anna Meyendorff and Anjan } \\
\text { Thakor. }\end{array}$} & $\begin{array}{l}\text { Lubomir Lizal, Miroslav Singer, } \\
\text { and Jan Svejnar }\end{array}$ & Dec. 1996 \\
\hline \multicolumn{2}{|c|}{$\begin{array}{l}\text { No. } 12 \text { Published in: Journal of International Marketing, "Executive } \\
\text { Insights: Marketing Issues and Challenges in Transitional Economies." } \\
\text { Vol. 5, No. 4, 1997, pp. 95-114. Also published in: Marketing Issues in } \\
\text { Transitional Economies ed. Rajeev Batra. }\end{array}$} & Rajeev Batra & Apr. 1997 \\
\hline \multicolumn{2}{|c|}{$\begin{array}{l}\text { No. 11: Worker Trust and System Vulnerability in the Transition from } \\
\text { Socialism to Capitalism }\end{array}$} & Andrew Schotter & Aug. 1996 \\
\hline \multicolumn{2}{|c|}{$\begin{array}{l}\text { No. } 10 \text { Published in: Comparative Economic Studies, "Russian Firms in } \\
\text { Transition: Champions, Challengers, and Chaff." Vol. 39, No.2, } \\
\text { Summer 1997, pp. 1-36. }\end{array}$} & Susan J. Linz & July 1996 \\
\hline \multicolumn{2}{|c|}{$\begin{array}{l}\text { No. 9: Corporate Debt Crisis and Bankruptcy Law During the } \\
\text { Transition: The Case of China }\end{array}$} & David D. Li and Shan Li & Dec. 1995 \\
\hline \multicolumn{2}{|c|}{$\begin{array}{l}\text { No. } 8 \text { Published in: Journal of Comparative Economics, "A Theory of } \\
\text { Ambiguous Property Rights in Transition Economies: The Case of the } \\
\text { Chinese Non-State Sector." Vol. 23, No. 1, Aug. 1996, pp. 1-19. }\end{array}$} & David D. Li & June 1996 \\
\hline \multicolumn{2}{|c|}{$\begin{array}{l}\text { No. 7: The Foreign Economic Contract Law of China: Cases and } \\
\text { Analysis }\end{array}$} & Dong-lai Li & June 1993 \\
\hline \multicolumn{2}{|c|}{$\begin{array}{l}\text { No. 3: Bank Privatization in Hungary and the Magyar Kulkereskedelmi } \\
\text { Bank Transaction }\end{array}$} & $\begin{array}{l}\text { Roger Kormendi and Karen } \\
\text { Schnatterly }\end{array}$ & May 1996 \\
\hline $\begin{array}{l}\text { Replacing No. 1: Journal of Comparative Economics } \\
\text { Symposium on "Bank Privatization in Central Europe and } \\
\text { Russia." Vol. 25, No. 1, Aug. } 1997 .\end{array}$ & \multicolumn{2}{|c|}{$\begin{array}{l}\text { "Bank Privatization in Transitional } \\
\text { Economies," Roger Kormendi and Edward } \\
\text { Snyder. }\end{array}$} & Aug. 1997 \\
\hline $\begin{array}{l}\text { Replacing No. 2: Journal of Comparative Economics } \\
\text { Symposium on "Bank Privatization in Central Europe and } \\
\text { Russia." Vol. 25, No. 1, Aug. } 1997 .\end{array}$ & \multicolumn{2}{|c|}{$\begin{array}{l}\text { "Transactional Structures of Bank } \\
\text { Privatizations in Central Europe and } \\
\text { Russia," Anna Meyendorff and Edward A. } \\
\text { Snyder. }\end{array}$} & Aug. 1997 \\
\hline $\begin{array}{l}\text { Replacing No. 4: Journal of Comparative Economics } \\
\text { Symposium on "Bank Privatization in Central Europe and } \\
\text { Russia." Vol. 25, No. 1, Aug. } 1997 .\end{array}$ & \multicolumn{2}{|c|}{$\begin{array}{l}\text { "Bank Privatization in Poland: The Case of } \\
\text { Bank Slaski," Jeffery Abarbaness and John } \\
\text { Bonin. }\end{array}$} & Aug. 1997 \\
\hline $\begin{array}{l}\text { Replacing No. 5: Journal of Comparative Economics } \\
\text { Symposium on "Bank Privatization in Central Europe and } \\
\text { Russia." Vol. 25, No. 1, Aug. } 1997 .\end{array}$ & \multicolumn{2}{|c|}{$\begin{array}{l}\text { "Bank Privatization in Post-Communist } \\
\text { Russia: The Case of Zhilsotsbank," Jeffery } \\
\text { Abarbanell and Anna Meyendorff }\end{array}$} & Aug. 1997 \\
\hline $\begin{array}{l}\text { Replacing No. 6: Journal of Comparative Economics } \\
\text { Symposium on "Bank Privatization in Central Europe and } \\
\text { Russia." Vol. 25, No. 1, Aug. } 1997 .\end{array}$ & \multicolumn{2}{|c|}{$\begin{array}{l}\text { "The Czech Republic's Commercial Bank: } \\
\text { Komercni Banka," Edward A. Snyder and } \\
\text { Roger C. Kormendi. }\end{array}$} & Aug. 1997 \\
\hline
\end{tabular}

